\title{
THE PARAMETERIZATION METHOD FOR INVARIANT MANIFOLDS II: REGULARITY WITH RESPECT TO PARAMETERS
}

\author{
XAVIER CABRÉ, ERNEST FONTICH, AND RAFAEL DE LA LLAVE
}

\begin{abstract}
We study the regularity with respect to parameters of the invariant manifolds associated to non-resonant subspaces obtained in the previous article [CFAILOO].
\end{abstract}

\section{Introduction AND STATEMENTS OF RESUlts}

This article is devoted to establish the regularity on parameters version of the results obtained in [CF(II)0] on existence and uniqueness of invariant manifolds associated to non-resonant subspaces. We refer to [CFdIL(0)] for the motivation, properties, notation, and references concerning such manifolds.

In the theory of dynamical systems, maps or equations usually appear depending on one or several parameters. It is then important to know the smooth dependence of dynamical objects with respect to the parameters. Moreover, results on regularity with respect to parameters are often needed when carrying out perturbation theories or transversality arguments.

The main results of this paper are Theorems 1.1] and [1.2, which are the parameter versions of Theorems 1.1 and 1.2 of [CFdIL(0)], respectively.

Throughout this paper we represent functions $G$ defined in subsets of Banach spaces $\Lambda_{0} \times X_{1}$, or of $\Lambda_{0} \times X$, by $G_{\lambda}(x)=G(\lambda, x)$. Therefore, we write $G, G_{\lambda}$ or $G_{\lambda}(x)$ according to the context. In particular, in formulas involving composition in the $x$ variable, where $\lambda$ acts as a parameter, we write $G_{\lambda} \circ H_{\lambda}$ for $G(\lambda, H(\lambda, \cdot))$.

Theorem 1.1. Let $X$ be a real or complex Banach space, $U$ an open set of $X, 0 \in$ $U$, let $\Lambda$ be an open subset of a Banach space $\Lambda_{0}$, and let $F: \Lambda \times U \subset \Lambda_{0} \times X \rightarrow X$, that we will write as $F_{\lambda}(x)$. Assume that $F_{\lambda}(0)=0$ and $F \in C^{r}(\Lambda \times U, X)$ for some $r \in \mathbb{N} \cup\{\infty, \omega\}$, i.e., $F$ is jointly $C^{r}$ in its two arguments.

Let $A_{\lambda}=D_{x} F_{\lambda}(0), N_{\lambda}(x)=F_{\lambda}(x)-A_{\lambda} x$, and $X=X_{1} \oplus X_{2}$ be a direct sum decomposition into closed subspaces independent of $\lambda$. Denote by $\pi_{1}, \pi_{2}$ the corresponding projections.

Assume that, uniformly for all $\lambda \in \Lambda$ :

0) $F_{\lambda}$ is a local diffeomorphism. In particular, $A_{\lambda}$ is invertible. 
1) The space $X_{1}$ is invariant under $A_{\lambda}$. That is,

$$
A_{\lambda} X_{1} \subset X_{1} \text {. }
$$

Let $A_{1, \lambda}=\left.\pi_{1} A_{\lambda}\right|_{X_{1}}, A_{2, \lambda}=\left.\pi_{2} A_{\lambda}\right|_{X_{2}}$ and $B_{\lambda}=\left.\pi_{1} A_{\lambda}\right|_{X_{2}}$. Hence, we have $A_{\lambda}=\left(\begin{array}{cc}A_{1, \lambda} & B_{\lambda} \\ 0 & A_{2, \lambda}\end{array}\right)$ with respect to the above decomposition. Assume:

2) $\operatorname{Spec}\left(A_{1, \lambda}\right) \subset\{z \in \mathbb{C}|| z \mid<1\}$.

3) $0 \notin \operatorname{Spec}\left(A_{2, \lambda}\right)$.

Let $L \geq 1$ be an integer independent of $\lambda$ such that

$$
\left(\operatorname{Spec}\left(A_{1, \lambda}\right)\right)^{L+1} \operatorname{Spec}\left(A_{\lambda}^{-1}\right) \subset\{z \in \mathbb{C}|| z \mid<1\},
$$

and assume that:

4) $\left(\operatorname{Spec}\left(A_{1, \lambda}\right)\right)^{i} \cap \operatorname{Spec}\left(A_{2, \lambda}\right)=\emptyset$ for every integer $i$ with $2 \leq i \leq L$ (in case that $L \geq 2$ ).

5) $L+1 \leq r$.

Then, we can find a map $R_{\lambda}: X_{1} \rightarrow X_{1}$ which is a polynomial in $x$ of degree not larger than $L$ with

$$
R_{\lambda}(0)=0, \quad D_{x} R_{\lambda}(0)=A_{1, \lambda},
$$

and a map $K_{\lambda}: U_{1} \subset X_{1} \rightarrow X$, where $U_{1}$ is an open neighborhood of 0 , such that

$$
F_{\lambda} \circ K_{\lambda}=K_{\lambda} \circ R_{\lambda}
$$

holds in $U_{1}$, and

$$
\begin{aligned}
& K_{\lambda}(0)=0, \\
& \pi_{1} D_{x} K_{\lambda}(0)=\mathrm{Id}, \quad \pi_{2} D_{x} K_{\lambda}(0)=0 .
\end{aligned}
$$

Moreover, $K$ is $C^{r-L-1}$ and $R$ is $C^{r-L}$ jointly in their two arguments.

Note that (2)-(5) guarantee that $K_{\lambda}\left(U_{1}\right)$ is an invariant manifold under $F_{\lambda}$, tangent to $X_{1}$ at the origin. We also recall that in [CFAlL00] we already proved the existence, for every fixed $\lambda$, of the polynomial $R_{\lambda}$ and of the map $K_{\lambda}$. We also established that $K_{\lambda}$ is $C^{r}$ in the $x$ variable.

Remark 1. When $r=\infty$ (that is, $F$ is $C^{\infty}$ with respect to both variables), Theorem 1.1] gives that the invariant manifold is also $C^{\infty}$ with respect to both variables. We will prove this result using the finite differentiability result of Theorem 1.1. The detailed proof is given in next section, following Lemma 2.2.

If $F$ is in the class $C^{\omega}$ of analytic maps with respect to both variables, then the invariant manifold is also analytic with respect to both variables. The proof in this case is much simpler than the finite differentiability result of Theorem 1.1. It can be obtained from an application of the implicit function theorem; see Remark [1]. 
Remark 2. In 1) of the previous theorem, the assumption that the invariant subspace $X_{1}$ of $A_{\lambda}$ does not depend on $\lambda$ is not a serious restriction. If the invariant subspace $X_{1, \lambda}$ depends smoothly on $\lambda$, one can make a change of coordinates in such a way that $X_{1, \lambda}$ becomes independent of $\lambda$, and then one can apply Theorem 1.1 to the transformed family of maps. Note that in case that $X_{1, \lambda} \oplus X_{2, \lambda}$ is a spectral decomposition, then the smooth dependence of $X_{i, \lambda}$ on $\lambda$ is a consequence of the spectral theorem.

As in the corresponding theorems of [CFAlL(00], the following result is stronger than Theorem 1.1, in the sense that hypothesis (6) below is weaker than (11). The differences in the conclusions of both results are the differentiability of $R$ and that, in the following theorem, $R_{\lambda}$ is no longer a polynomial in $x$.

Theorem 1.2. Assume hypotheses 0) - 5) of Theorem 1.1 hold uniformly for all $\lambda \in \Lambda$, except that (1) is replaced by

$$
\left(\operatorname{Spec}\left(A_{1, \lambda}\right)\right)^{L+1} \operatorname{Spec}\left(A_{2, \lambda}^{-1}\right) \subset\{z \in \mathbb{C}|| z \mid<1\}, \quad L \geq 1 .
$$

Then, we can find maps $K_{\lambda}$ and $R_{\lambda}$ which are $C^{r-L-1}$ jointly in their two arguments, satisfy (3),

$$
K_{\lambda}(0)=0, \quad \pi_{1} K_{\lambda}=\mathrm{Id}, \quad \pi_{2} D_{x} K_{\lambda}(0)=0,
$$

and

$$
R_{\lambda}(0)=0, \quad D_{x} R_{\lambda}(0)=A_{1, \lambda} .
$$

We refer to [CFAIL00] for uniqueness statements and for further properties of the maps $K_{\lambda}$ and $R_{\lambda}$ in the two previous theorems.

Theorems 1.1 and 1.2 will follow from sharper regularity results, Theorems 2.1 and 3.1 below, that provide optimal regularity in a certain class -given by Definition 1.3 of differentiable maps of two Banach space variables. We will prove that, in such a class, the parameterization $K$ has the same regularity as the map $F$ (that is, no derivative is lost). Since these classes include $C^{r}$ maps, we will deduce the two previous theorems.

Recall that the starting point of our proofs consists of finding maps $R_{\lambda}$ and $K_{\lambda}^{\leq}$which are polynomials in $x$ of degree $L$, and satisfy the functional equation $F_{\lambda} \circ K_{\lambda}=K_{\lambda} \circ R_{\lambda}$ up to order $L$ at the origin. We then write

$$
K=K_{\lambda}^{\leq}+K^{>}=K_{\lambda}^{\leq}+K_{\lambda}^{>}
$$

and we study the functional equation (3) for $K^{>}$.

Remark 3. If we try to use the implicit function theorem to solve (3) for $K^{>}$,

$$
F_{\lambda} \circ\left(K_{\lambda}^{\leq}+K^{>}\right)=\left(K_{\lambda}^{\leq}+K^{>}\right) \circ R_{\lambda}
$$

we are led to consider the operator defined by

$$
\mathcal{P}\left(\lambda, K^{>}\right)=F_{\lambda} \circ\left(K_{\lambda}^{\leq}+K^{>}\right)-\left(K_{\lambda}^{\leq}+K^{>}\right) \circ R_{\lambda} .
$$

By [CFAlLO0] we already know that under the hypotheses of Theorem 1.1, for each $\lambda_{0}$ there exists $K_{\lambda_{0}}^{>}$such that $\mathcal{P}\left(\lambda_{0}, K_{\lambda_{0}}^{>}\right)=0$. 
Here we are interested in the regularity of $K_{\lambda}^{>}$with respect to $\lambda$. The following technical difficulty appears. The map $\left(\lambda, K^{>}\right) \mapsto K^{>} \circ R_{\lambda}$, where $K^{>}=K^{>}(x)$ is a function of $x$ alone, can not be differentiable from $\Lambda \times C^{r}\left(X_{1}\right)$ to $C^{r}\left(X_{1}\right)$ for any $r \in \mathbb{N}$. Indeed, the derivative of this map with respect to $\lambda$ should be $K^{>} \mapsto D K^{>} \circ R_{\lambda} D_{\lambda} R_{\lambda}$, which maps $C^{r}$ into $C^{r-1}$, but not into $C^{r}$. Therefore, it is not possible to apply the implicit function theorem in a straightforward way.

The situation changes if it happens that $R$ does not depend on $\lambda$. For instance, this is the case when $A_{1, \lambda}=A_{1}$ does not depend on $\lambda$ (that is, only the nonlinear terms depend on $\lambda$ ) and the non-resonance assumptions

$$
\left(\operatorname{Spec}\left(A_{1}\right)\right)^{i} \cap \operatorname{Spec}\left(A_{1}\right)=\emptyset \quad \text { for every integer } i \text { with } 2 \leq i \leq L,
$$

of Theorem 1.1 in [CFAlLO0], are satisfied. In such case, $R$ can be taken to be equal to $A_{1}$ (in particular, to be independent of $\lambda$ ), and one can obtain regularity results very quickly using the implicit function theorem. However, the results obtained applying the implicit function theorem to $\mathcal{P}$ in (7) are always one derivative short from optimal. The use of the implicit function theorem is presented in an expository way in one of the sections of [CFdILO2].

If $F$ is analytic, then the map $\mathcal{P}$ in (77) is differentiable in the appropriate spaces of analytic functions for $K^{>}$considered in [CFdILO0]. This holds in the general case when $R$ depends on $\lambda$. From this, one can deduce the analytic dependence on $\lambda$ of the solution $K^{>}$of $\mathcal{P}=0$; see [CFdIL02] for more details.

The method of proof of Theorem 1.1 follows closely the one of optimal regularity in Theorem [1.1 of [CFdIL00]. That is, we study the functional equation for $K^{>}$as a fixed point problem, and we show that the corresponding operator $\mathcal{T}$ is a contraction in a suitable class of differentiable functions. In this way, we obtain a map $K^{>}$which has almost optimal regularity with respect to the variables $(\lambda, x)$. Then, studying an equation satisfied by the derivative $D_{x} K^{>}$, we get the optimal regularity for $K^{>}$stated in Theorem 1.1 (see Section 2).

This kind of proof does not work for Theorem 1.2, since the parameter version of the fixed point operator $\mathcal{M}$ corresponding to Theorem 1.2 is not a contraction in the usual norms of spaces of differentiable functions.

The proof of Theorem 1.2 follows a different route. We inductively prove that the derivatives in the parameter and in the variable $x$ exist and are continuous and bounded, provided that derivatives of "lower order" exist and are continuous and bounded. In the beginning of Section 31 we describe the proof and then, in the same section, we present all its details.

In both Theorems [1.1 and [1.2, the precise definition of derivatives of lower order is somewhat intricate (they are derivatives in two variables, so the ordering has to involve two indices) and will be motivated by the induction proof and the structure of the operators, which involve composition in the $x$ variable. That is, we will define a suitable order on the sets of indices of derivatives for functions of two variables, in such a way that we can use the functional equation to express 
derivatives of a certain index in terms of derivatives with indices which are smaller in the indicated order.

We now present the classes of differentiable functions that we will use. We consider functions $f: \Lambda \times U \rightarrow Y$, where $\Lambda$ and $U$ are open sets in two Banach spaces, and $Y$ is another Banach space.

We say that $f$ is $C^{l, m}$ when $D_{\lambda}^{i} D_{x}^{j} f(\lambda, x)$ exist, are continuous and bounded for $0 \leq i \leq l, 0 \leq j \leq m$. We consider the space of such functions endowed with the topology given by the supremum of all the derivatives above.

We say that $f$ is jointly $C^{r}$ when it is a $C^{r}$ mapping from $\Lambda \times U \rightarrow Y$ with bounded derivatives up to order $r$. This is equivalent to the existence, continuity and boundedness of $D_{\lambda}^{i} D_{x}^{j} f(\lambda, x)$ for $0 \leq i+j \leq r$.

More generally, if $\Sigma \subset\left(\mathbb{Z}^{+}\right)^{2}$ is such that $(i, j) \in \Sigma$ and $\tilde{\imath} \leq i, \tilde{\jmath} \leq j$ implies $(\tilde{\imath}, \tilde{\jmath}) \in \Sigma$, we denote by $C^{\Sigma}$ the set of functions $f$ for which $D_{\lambda}^{i} D_{x}^{j} f$ exists, is continuous and bounded for every $(i, j) \in \Sigma$. We consider $C^{\Sigma}$ endowed with the norm

$$
\|f\|_{C^{\Sigma}(B)}=\sup _{(i, j) \in \Sigma,(\lambda, x) \in B}\left|D_{\lambda}^{i} D_{x}^{j} f(\lambda, x)\right|,
$$

which makes it a Banach space. When the set $B$ is understood from the context, we will suppress it from the notation.

It is clear that if $\Sigma^{\prime} \subset \Sigma$ then $C^{\Sigma} \subset C^{\Sigma^{\prime}}$. In particular, $C^{l+r} \subset C^{l, r}$, $C^{r, r} \subset C^{r}, C^{l+1, r} \subset C^{l, r}$ and $C^{l, r+1} \subset C^{l, r}$. Moreover, except for trivial cases, these inclusions are strict.

Now we turn to the definition of derivatives of "lower order". The operators $\mathcal{T}$ and $\mathcal{M}$ involve compositions. If $f_{\lambda}$ and $g_{\lambda}$ are families of maps, when taking derivatives of order $i$ of the composition $h_{\lambda}=f_{\lambda} \circ g_{\lambda}=f_{\lambda}\left(\lambda, g_{\lambda}(\lambda, \cdot)\right)$ with respect to parameters, we are forced to take the same number $i$ of derivatives of $f_{\lambda}$ with respect to the variable $x$, and not only with respect to the parameter. Quantitative versions of this fact are made precise in Lemmas [2.3 and 3.6 below.

The previous consideration leads naturally to the following class of differentiable functions. It will be well adapted to the study of regularity with respect to parameters.

Definition 1.3. Given $(i, j) \in\left(\mathbb{Z}^{+}\right)^{2}$, we define

$$
\Sigma_{i, j}=\left\{(a, b) \in\left(\mathbb{Z}^{+}\right)^{2} \mid a+b \leq i+j \text { and } a \leq i\right\} .
$$

We say that the derivative $D_{\lambda}^{a} D_{x}^{b}$ is of lower order than the derivative $D_{\lambda}^{i} D_{x}^{j}$ if $(a, b) \in \Sigma_{i, j}$.

It is easy to verify that being of "lower order than" is a partial order relation. Note also that $C^{\Sigma_{r, 0}}=C^{r}$.

\section{Proof of Theorem [T.]}

The following is the main result of this section. It is formulated in the classes $C^{\Sigma_{i, j}}$ which generalize the $C^{r}$ class. 
We assume that $j \geq L+1$ and that $F$ has derivatives $D_{\lambda}^{a} D_{x}^{b} F$ for each $(a, b)$ in the set $\Sigma_{i, j}$. We establish that the parameterization $K$ given by Theorem 1.1 of [CFdIL00] has derivatives $D_{\lambda}^{a} D_{x}^{b} K$ for each $(a, b)$ in the same set $\Sigma_{i, j}$. We point out that here we do not assume $F$ to be jointly $C^{r}$ in its two arguments. We will see that Theorem 1.1 follows easily from this result.

Theorem 2.1. Let $\Lambda_{0}$ and $X$ be Banach spaces, $\Lambda$ an open set of $\Lambda_{0}$, and $U$ an open set of $X$ with $0 \in U$. Let $F: \Lambda \times U \longrightarrow X$ be a map such that:

1) For some $(i, j) \in\left(\mathbb{Z}^{+}\right)^{2}$,

$$
F \in C^{\Sigma_{i, j}}
$$

where $\Sigma_{i, j}$ is the set introduced in Definition 1.3, and $C^{\Sigma_{i, j}}$ denotes the set of functions which have all the derivatives of orders in $\Sigma_{i, j}$ defined, continuous and bounded.

2) Conditions 0)-4) of Theorem 1.1 are satisfied.

3) $L+1 \leq j$.

Then, we can find maps $K \in C^{\Sigma_{i, j}}$ and $R \in C^{i, \infty} \subset C^{\Sigma_{i, j}}$ satisfying all the properties stated in Theorem 1.1 .

To prove the previous result, and also Theorem 1.1, we start by studying the regularity with respect to $\lambda$ of the polynomial maps $K_{\lambda}^{\leq}$and $R_{\lambda}$. This is a simple task. Indeed, we follow the proof of Lemma 3.1 in [CFdILO0]. We find polynomials $K^{\leq}=\sum_{m=1}^{L} K_{m}$ and $R=\sum_{m=1}^{L} R_{m}$, with $K_{m}$ and $R_{m}$ satisfying (see [CFAIL00] for the notation)

$$
\begin{aligned}
& \left(\mathcal{L}_{A_{1, \lambda}}-\mathcal{L}_{m, A_{1, \lambda}, \text { Id }}\right) K_{m}^{1}-R_{m}=-\Gamma_{m}^{1}-B K_{m}^{2} \\
& \left(\mathcal{L}_{A_{2, \lambda}}-\mathcal{L}_{m, A_{1, \lambda}, \text { Id }}\right) K_{m}^{2}=-\Gamma_{m}^{2}
\end{aligned}
$$

making the same choice (for all the values of $\lambda$ ) on which terms of equations (8) and (9) are eliminated. In this way we get that, under the assumptions of Theorem 2.1, $K_{\lambda}^{\leq}$and $R_{\lambda}$ are polynomials in $x$ of degree $L$, and of class $C^{i}$ with respect to $\lambda$.

To justify the previous statement, note that the mappings that to $A_{1, \lambda}, A_{2, \lambda}$ associate $\mathcal{L}_{m, A_{1, \lambda}, A_{2, \lambda}}$ are analytic (in fact polynomial). Therefore, if $A_{1, \lambda}, A_{2, \lambda}$ are $C^{i}$ in $\lambda$, then

$$
\mathcal{L}_{A_{1, \lambda}}, \mathcal{L}_{A_{2, \lambda}}, \mathcal{L}_{m, A_{1, \lambda}, A_{2, \lambda}^{-1}} \text { and } \mathcal{L}_{m, A_{1, \lambda}, A_{1, \lambda}^{-1}}
$$

are also $C^{i}$ as maps from $\Lambda$ to $L\left(\mathbf{M}_{m}, \mathbf{M}_{m}\right)$. Therefore, if the right hand sides of (8) and (9) are $C^{i}$ in $\lambda$, then $K_{m}^{1}, K_{m}^{2}$ and $R_{m}$ are also $C^{i}$ in $\lambda$.

For further reference, we summarize the previous argument in the following:

Lemma 2.2. Assume that $F \in C^{\Sigma_{i, j}}$ for some $j \geq L$, and that hypotheses 0 ), 1), 3) and 4) of Theorem 1.1 are satisfied uniformly in $\lambda$. Then, 
a) We can find polynomials in $x, K_{\lambda}^{\leq}=\sum_{m=1}^{L} K_{m}$ and $R_{\lambda}=\sum_{m=1}^{L} R_{m}$, of degree not larger than $L$, where $K_{m}$ and $R_{m}$ are homogeneous polynomials of degree $m$, satisfying

$$
F_{\lambda} \circ K_{\lambda}^{\leq}(x)=K_{\lambda}^{\leq} \circ R_{\lambda}(x)+o\left(|x|^{L}\right),
$$

and (2), (四), (5), i.e., $R_{\lambda}(0)=0, D_{x} R_{\lambda}(0)=A_{1, \lambda}, K_{\lambda}^{\leq}(0)=0$, and $D_{x} K_{\lambda}^{\leq}(0)=(\mathrm{Id}, 0)$.

b) If we further assume that

$$
\left(\operatorname{Spec}\left(A_{1, \lambda}\right)\right)^{j} \cap \operatorname{Spec}\left(A_{1, \lambda}\right)=\emptyset \quad \text { for some } j \text { with } 2 \leq j \leq L,
$$

then we can choose $R_{j}=0$.

c) The maps $K^{\leq}$and $R$ belong to $C^{i, \infty}$. That is, $K_{\lambda}^{\leq}$and $R_{\lambda}$ are polynomials in $x$ of class $C^{i}$ with respect to $\lambda$.

For every given $\lambda \in \Lambda$, the parameterization $K_{\lambda}^{>}$given by Theorem 1.1 of [CFAIL00] is the fixed point of the operator $\mathcal{T}_{\lambda}$ defined by

$$
\mathcal{T}_{\lambda} K_{\lambda}^{>} \equiv \mathcal{S}_{\lambda}^{-1} \tilde{H}_{\lambda}=\sum_{k=0}^{\infty} A_{\lambda}^{-(k+1)} \tilde{H}_{\lambda} \circ R_{\lambda}^{k},
$$

where, for $K_{\lambda}^{>}$given, $\tilde{H}_{\lambda}$ is defined by

$$
\tilde{H}_{\lambda}=-N_{\lambda} \circ\left(K_{\lambda}^{\leq}+K_{\lambda}^{>}\right)-A_{\lambda} K_{\lambda}^{\leq}+K_{\lambda}^{\leq} \circ R_{\lambda},
$$

and $K_{\lambda}^{\leq}$and $R_{\lambda}$ are the polynomials of degree $L$ given by Lemma 2.2 .

To prove Theorem 2.1, we scale $F$ with respect to both variables. More precisely, given $\lambda_{0} \in \Lambda$, we consider the family

$$
\tilde{F}_{\lambda}(x)=\delta^{-1} F_{\lambda_{0}+\delta \lambda}(\delta x)
$$

where $\delta>0$. The same expression (13) holds with $F_{\lambda}$ replaced by $N_{\lambda}$, the nonlinear part of the map $F_{\lambda}$. Recall that $N_{\lambda}(0)=D_{x} N_{\lambda}(0)=0$ for every $\lambda \in \Lambda$. Hence we also have $D_{\lambda} N_{\lambda}(0)=0$ for every $\lambda \in \Lambda$. In particular, $N, D_{x} N$ and $D_{\lambda} N$ all vanish at the point $\left(\lambda_{0}, 0\right)$. Therefore, using Taylor's theorem in the expression (13) for $\tilde{N}$, taking $\delta$ small (and renaming the corresponding $\tilde{N}$ by $N)$, we can assume that

$$
\|N\|_{C^{\Sigma_{i, j}\left(\Lambda \times B_{1}\right)}},\left\|K^{\leq}-\mathrm{Id}\right\|_{C^{i, j}\left(\Lambda \times B_{1}\right)} \text {, and }\left\|R-A_{1, \lambda}\right\|_{C^{i, j}\left(\Lambda \times B_{1}\right)}
$$

are as small as we need.

Proof of Theorem 1.1. We first consider the case $r \in \mathbb{N}$. We assume that $F$ is $C^{r}$ jointly in its two arguments, and that $r \geq L+1$. Since $F \in C^{r} \subset C^{\Sigma_{r-L, L}}$, Lemma 2.2 applied with $i=r-L$ and $j=L$ gives that $R \in C^{r-L, \infty}$, and hence $R$ is $C^{r-L}$ jointly in its two variables. Regarding the parameterization $K$, note that $F \in C^{r} \subset C^{\Sigma_{r-L-1, L+1}}$. Applying Theorem 2.1, we conclude that $K \in C^{\Sigma_{r-L-1, L+1}} \subset C^{r-L-1}$.

The case when $r=\infty$ follows from the finite differentiability case. The argument is very similar to the corresponding one in [CFdIL00]. The key point 
is to show that when the function $F$ is $C^{\infty}$, all the finite differentiable solutions obtained by applying Theorem 2.1 agree.

This is not completely obvious since the proof of Theorem 2.1 depends on scaling the initial problem and the scaling depends on the degree of differentiability required - recall (13) and condition (14). So that, even if a direct application of Theorem 2.1] provides uniqueness in a small neighborhood for each finite regularity - they are based on a contraction mapping principle - we will need to discuss the possibility that the neighborhoods shrink to just the origin as the regularity increases. Indeed, we recall that this phenomenon happens for center manifolds.

There are several observations that allow us to obtain the desired uniqueness and, therefore, the case $r=\infty$ from the finite differentiable cases.

First, we note that, once we fix $r$, all the non-resonance conditions up to order $r$ hold uniformly for $\lambda \in \Lambda$. In particular, once we fix the procedure to choose the $K \leq$ and the $R$, we can assume that the $K \leq$ and $R$ are uniformly regular and that, since they are polynomials of degree $L$ determined by the jets, they are independent of $r$ provided that $r>L$. We can also assume that $R_{\lambda}$ is contraction in $U_{1}$ uniformly in $\lambda \in \Lambda$.

Second, we observe that, as in the proof of the corresponding theorem in [CFAII00], if we fix $\lambda$, the functional equation (3), $F_{\lambda} \circ K_{\lambda}=K_{\lambda} \circ R_{\lambda}$, leads to

$$
K_{\lambda}=F_{\lambda}^{-j} \circ K_{\lambda} \circ R_{\lambda}^{j} \quad \text { for every } j \geq 1 .
$$

Therefore, since $R_{\lambda}$ is a uniform contraction, equation (15) (used with $j$ large) allows to recover $K_{\lambda}$ in $U_{1}$ from $K_{\lambda}$ restricted to any smaller neighborhood of the origin in $X_{1}$. In particular, if we fix $\lambda$, two $K_{\lambda}$ that solve the equation (3) and agree in a open neighborhood of the origin in $X_{1}$, will also agree in $U_{1}$.

Now, for $\lambda_{0} \in \Lambda$, we consider the scaled family (13). By choosing $\delta$ small enough - depending on $r$ - we can ensure that the smallness conditions (14) are satisfied. Therefore, we obtain a $K$ defined on $B_{\rho}\left(\lambda_{0}\right) \times B_{\mu}(0)$. The positive numbers $\rho$ and $\mu$ could depend on $r$. We will denote them as $\rho(r), \mu(r)$ to emphasize this fact.

If we now consider the solution for another $r^{\prime}>r$, we know that they have to agree on $B_{\rho\left(r^{\prime}\right)}\left(\lambda_{0}\right) \times B_{\mu\left(r^{\prime}\right)}(0)$. Now, as we mentioned above, using equation (15) we obtain that they agree on $B_{\rho\left(r^{\prime}\right)}\left(\lambda_{0}\right) \times U_{1}$.

Since $\lambda_{0}$ is arbitrary, we obtain that any solution that is $C^{r}$ in $\Lambda \times U_{1}$ must be $C^{r^{\prime}}$ as well. This finishes the proof of the $r=\infty$ case.

Remark 4. Note that using equation (3) to improve the domain of uniqueness of the parameterization $K$ is the step that is missing in the case of center manifolds.

Notice also that the uniqueness holds only once we have specified the algorithm to obtain the $K \leq$ and the $R$. In this case, the uniqueness and the differentiablity is clear since $K \leq$ and $R$ are polynomials that are obtained from the $L$-jet of $F$ by applying a finite number of operations that clearly yield smoothness. 
The scheme of the proof of Theorem 2.1 is the same as in the proofs of existence and sharp regularity of $K$ in [CFdILO0]. The main difference is that here we will work in the following spaces of differentiable functions of two Banach space variables.

For $s \geq l$, let

$$
\begin{aligned}
\|H\|_{\Gamma_{i, s, l}}:=\max \left\{\max _{(a, b) \in \Sigma_{i, s}}\left\|D_{\lambda}^{a} D_{x}^{b} H\right\|_{C^{0}\left(\Lambda \times B_{1}\right)},\right. \\
\left.\max _{0 \leq a \leq i} \sup _{(\lambda, x) \in \Lambda \times B_{1}}\left(\left|D_{\lambda}^{a} D_{x}^{l} H_{\lambda}(x)\right| /|x|\right)\right\}
\end{aligned}
$$

and

$$
\begin{aligned}
\Gamma_{i, s, l}= & \left\{H: \Lambda \times B_{1} \subset \Lambda_{0} \times X_{1} \longrightarrow Y \mid H \in C^{\Sigma_{i, s}}\left(\Lambda \times B_{1}\right),\right. \\
& \left.D_{\lambda}^{a} D_{x}^{b} H_{\lambda}(0)=0 \text { if } 0 \leq a \leq i \text { and } 0 \leq b \leq l,\|H\|_{\Gamma_{i, s, l}}<\infty\right\} .
\end{aligned}
$$

The norm $\|\cdot\|_{\Gamma_{i, s, l}}$ makes $\Gamma_{i, s, l}$ a Banach space. Note also that the terms $\sup _{(\lambda, x) \in \Lambda \times B_{1}}\left(\left|D_{\lambda}^{a} D_{x}^{l} H_{\lambda}(x)\right| /|x|\right)$ included in the definition of the norm $\|\cdot\|_{\Gamma_{i, s, l}}$ are relevant only when $s=l$, in the sense that we could omit them when $s>l$ and still get an equivalent norm (since $D_{\lambda}^{a} D_{x}^{l} H_{\lambda}(0)=0$ ).

Note that if $H \in \Gamma_{i, s, l}$ and $(a, b) \in \Sigma_{i, s}$, using Taylor's theorem and the definition of $\|\cdot\|_{\Gamma_{i, j, l}}$, we have

$$
\left|D_{\lambda}^{a} D_{x}^{b} H_{\lambda}(x)\right| \leq \frac{1}{(l-b+1)_{+} !}\|H\|_{\Gamma_{i, s, l}}|x|^{(l-b+1)_{+}}, \quad(\lambda, x) \in \Lambda \times B_{1} .
$$

To describe in some detail the structure of the expression for the derivatives of the composition in terms of the derivatives of the functions, we introduce the following auxiliary sets of indices, closely related to $\Sigma_{i, j}$ :

$$
\begin{aligned}
& \Sigma_{i, 0}^{*}=\left\{(a, b) \in\left(\mathbb{Z}^{+}\right)^{2} \mid a+b \leq i, b \geq 1\right\} \cup\{(i, 0)\}, \\
& \Sigma_{i, j}^{*}=\left\{(a, b) \in\left(\mathbb{Z}^{+}\right)^{2} \mid a+b \leq i+j, a \leq i, b \geq 1\right\} \quad \text { if } j \geq 1,
\end{aligned}
$$

and

$$
\begin{aligned}
& \tilde{\Sigma}_{i, 0}=\left\{(a, b) \in\left(\mathbb{Z}^{+}\right)^{2} \mid a+b \leq i\right\}, \\
& \tilde{\Sigma}_{i, j}=\Sigma_{i, j}^{*} \quad \text { if } j \geq 1 .
\end{aligned}
$$

2.1. Solution of the fixed point problem. The following three lemmas will prove the existence of a solution $K^{>}$with almost optimal regularity, more precisely a solution $K^{>} \in C^{\Sigma_{i, j-1}}$.

Lemma 2.3. With the notation above, assuming that $H \in C^{\Sigma_{i, j}}, R \in C^{i, j}$ and $A_{\lambda} \in C^{i, \infty}$, we have that

$$
D_{\lambda}^{i} D_{x}^{j}\left[A_{\lambda}^{-(k+1)} H_{\lambda} \circ R_{\lambda}^{k}\right]
$$

is a polynomial expression that involves only derivatives $D_{\lambda}^{m} A_{\lambda}^{-(k+1)}$ with indices $0 \leq m \leq i$, derivatives $D_{\lambda}^{a} D_{x}^{b} H_{\lambda}$ evaluated at $R_{\lambda}^{k}$ with $(a, b) \in \tilde{\Sigma}_{i, j}$, and derivatives $D_{\lambda}^{\alpha} D_{x}^{\beta} R_{\lambda}^{k}$ with $0 \leq \alpha \leq i, 0 \leq \beta \leq j$, except for $(\alpha, \beta)=(0,0)$. 
Moreover, for each term in the polynomial expression there exist indices $0 \leq m \leq i,(a, b) \in \tilde{\Sigma}_{i, j}$ with $a \leq m$, and $\left(i_{l}, j_{l}\right)$ with $j_{1}+\cdots+j_{b}=j, i_{1}+\cdots+i_{b}=$ $m-a$ and $\left(i_{l}, j_{l}\right) \neq(0,0)$ for $1 \leq l \leq b$, such that the given term contains:

- the factor $D_{\lambda}^{i-m} A_{\lambda}^{-(k+1)}$,

- the factor $\left(D_{\lambda}^{a} D_{x}^{b} H_{\lambda}\right) \circ R_{\lambda}^{k}=D_{\lambda}^{a} D_{x}^{b} H_{\lambda} \circ R_{\lambda}^{k}$, and

- the factor $D_{\lambda}^{i_{1}} D_{x}^{j_{1}} R_{\lambda}^{k} \cdots D_{\lambda}^{i_{b}} D_{x}^{j_{b}} R_{\lambda}^{k}$.

Proof. We start by computing the $m^{\text {th }}$ derivative of $H_{\lambda} \circ R_{\lambda}^{k}$ with respect to $\lambda$. Let $T_{m}=D_{\lambda}^{m}\left[H_{\lambda} \circ R_{\lambda}^{k}\right]$. It is easily checked by induction that

$$
T_{m}=\sum_{(a, b) \in \Sigma_{m, 0}^{*}} \sum_{i_{1}, \ldots, i_{b}} C D_{\lambda}^{a} D_{x}^{b} H_{\lambda} \circ R_{\lambda}^{k} D_{\lambda}^{i_{1}} R_{\lambda}^{k} \cdots D_{\lambda}^{i_{b}} R_{\lambda}^{k},
$$

where the second sum runs over $i_{1}+\cdots+i_{b}=m-a$, and $C$ is a coefficient which depends on $a, b, i_{1}, \ldots, i_{b}$.

Then, we differentiate the expression (20) $j$ times with respect to $x$. It is also easy to see by induction that

$$
D_{x}^{j} T_{m}=\sum_{(a, b) \in \Sigma_{m, j}^{*} i_{1}, j_{1}, \ldots, i_{b}, j_{b}} C D_{\lambda}^{a} D_{x}^{b} H_{\lambda} \circ R_{\lambda}^{k} D_{\lambda}^{i_{1}} D_{x}^{j_{1}} R_{\lambda}^{k} \cdots D_{\lambda}^{i_{b}} D_{x}^{j_{b}} R_{\lambda}^{k},
$$

where the second sum runs over $i_{1}+\cdots+i_{b}=m-a, j_{1}+\cdots+j_{b}=j$, and $C$ is a combinatorial coefficient depending on the indices.

Finally, by Leibnitz formula,

$$
D_{\lambda}^{i} D_{x}^{j}\left[A_{\lambda}^{-(k+1)} H_{\lambda} \circ R_{\lambda}^{k}\right]=\sum_{m=0}^{i} C\left[D_{\lambda}^{i-m} A_{\lambda}^{-(k+1)}\right] D_{x}^{j} T_{m} .
$$

Substituting (21) into (22), the resulting expression contains derivatives $D_{\lambda}^{a} D_{x}^{b} H_{\lambda}$ of orders $(a, b) \in \Sigma_{0, j}^{*} \cup \Sigma_{1, j}^{*} \cup \cdots \cup \Sigma_{i, j}^{*}=\Sigma_{i, j}^{*}=\tilde{\Sigma}_{i, j}$ if $j>0$, or $\{(a, b) \mid a+b \leq i\}=\tilde{\Sigma}_{i, 0}$ if $j=0$.

Lemma 2.4. Let $R$ be the map obtained in Lemma 2.2. Assuming that $R \in$ $C^{i, \infty}, A_{\lambda} \in C^{i, \infty}$ and that $j \geq L$, then $\mathcal{S}: \Gamma_{i, j, L} \longrightarrow \Gamma_{i, j, L}$ defined by $(\mathcal{S} H)_{\lambda}=$ $A_{\lambda} H_{\lambda}-H_{\lambda} \circ R_{\lambda}$ is an invertible operator, and $\left\|\mathcal{S}^{-1}\right\|$ can be bounded by a constant independent of the scaling parameter.

Proof. The formal expression for $\mathcal{S}^{-1}$ is the parameter version of the one we have obtained in [CFAII(0)]:

$$
\left(\mathcal{S}^{-1} \eta\right)_{\lambda}=\sum_{k=0}^{\infty} A_{\lambda}^{-(k+1)} \eta_{\lambda} \circ R_{\lambda}^{k}
$$

We will show that the series (23) converges in the norm of $\Gamma_{i, j, L}$, and that

$$
\left\|\sum_{k=0}^{\infty} A_{\lambda}^{-(k+1)} \eta_{\lambda} \circ R_{\lambda}^{k}\right\|_{\Gamma_{i, j, L}} \leq C\|\eta\|_{\Gamma_{i, j, L}}
$$


We begin by showing that if $\eta \in \Gamma_{i, j, L}$ and $(a, b) \in \Sigma_{i, j}$ then the series

$$
\sum_{k=0}^{\infty} D_{\lambda}^{a} D_{x}^{b}\left[A_{\lambda}^{-(k+1)} \eta_{\lambda} \circ R_{\lambda}^{k}\right](x)
$$

converges absolutely. As in [CFAlL00] we will use the notation $(t)_{+}=\max (t, 0)$.

It is not hard to prove that

$$
\left\|D_{\lambda}^{m} A_{\lambda}^{-(k+1)}\right\| \leq C_{m}(k+1)^{m}\left\|A_{\lambda}^{-1}\right\|^{k+1}
$$

and that

$$
\left|D_{\lambda}^{m} D_{x}^{l} R_{\lambda}^{k}(x)\right| \leq C_{m, l}\left(\left\|A_{1, \lambda}\right\|+\varepsilon\right)^{k}|x|^{(1-l)_{+}}
$$

(these bounds are established in page 574 of [dILMM86]). By Lemma 2.3 every term in the sum (24) is bounded by a sum of terms of the form

$$
C\left\|D_{\lambda}^{a-m} A_{\lambda}^{-(k+1)}\right\|\left|D_{\lambda}^{\alpha} D_{x}^{\beta} \eta_{\lambda}\left(R_{\lambda}^{k}(x)\right)\right|\left|D_{\lambda}^{i_{1}} D_{x}^{j_{1}} R_{\lambda}^{k}(x)\right| \cdots\left|D_{\lambda}^{i_{\beta}} D_{x}^{j_{\beta}} R_{\lambda}^{k}(x)\right|
$$

with $0 \leq m \leq a,(\alpha, \beta) \in \tilde{\Sigma}_{m, b}, i_{1}+\cdots+i_{\beta}=m-\alpha$ and $j_{1}+\cdots+j_{\beta}=b$. Hence, every term in the sum (24) is bounded by

$$
\begin{gathered}
C(k+1)^{a-m}\left\|A_{\lambda}^{-1}\right\|^{k+1}\|\eta\|_{\Gamma_{i, j, L}}\left|R_{\lambda}^{k}(x)\right|^{(L-\beta+1)_{+}}\left(\left\|A_{1, \lambda}\right\|+\varepsilon\right)^{k \beta}|x|^{(\beta-b)_{+}} \\
\leq C\|\eta\|_{\Gamma_{i, j, L}}(k+1)^{a}\left\|A_{\lambda}^{-1}\right\|^{k+1}\left(\left\|A_{1, \lambda}\right\|+\varepsilon\right)^{k(L-\beta+1)_{+}}|x|^{(L-\beta+1)_{+}} \\
\cdot\left(\left\|A_{1, \lambda}\right\|+\varepsilon\right)^{k \beta}|x|^{(\beta-b)_{+}} \\
\leq C\|\eta\|_{\Gamma_{i, j, L}}(k+1)^{a}\left(\left\|A_{\lambda}^{-1}\right\|\left(\left\|A_{1, \lambda}\right\|+\varepsilon\right)^{L+1}\right)^{k}|x|^{(L-b+1)_{+}}
\end{gathered}
$$

with $C$ independent of $k$. We have used that

$$
\begin{gathered}
\left(1-j_{1}\right)_{+}+\left(1-j_{2}\right)_{+}+\cdots+\left(1-j_{\beta}\right)_{+} \geq\left(\beta-\left(j_{1}+\cdots+j_{\beta}\right)\right)_{+}=(\beta-b)_{+}, \\
(L-\beta+1)_{+}+\beta \geq L+1 \\
(L-\beta+1)_{+}+(\beta-b)_{+} \geq(L-b+1)_{+}
\end{gathered}
$$

which hold because the function $(\cdot)_{+}$is subadditive.

Since

$$
\sum_{k=1}^{\infty}(k+1)^{a}\left(\left\|A_{\lambda}^{-1}\right\|\left(\left\|A_{1, \lambda}\right\|+\varepsilon\right)^{L+1}\right)^{k}
$$

converges, we deduce that (24) converges absolutely in $\Lambda \times B_{1}$.

Hence, in the same way as in Lemma 3.3 in, we conclude that the expression (23) defines a function $H$ in $C^{\Sigma_{i, j}}$ which satisfies $\mathcal{S} H=\eta$. Moreover

$$
\left|D_{\lambda}^{a} D_{x}^{b}\left(\mathcal{S}^{-1} \eta\right)_{\lambda}(x)\right| \leq C\|\eta\|_{\Gamma_{i, j, L}}|x|^{(L-b+1)_{+}},
$$

and then, clearly, $\left\|\mathcal{S}^{-1} \eta\right\|_{\Gamma_{i, j, L}} \leq C\|\eta\|_{\Gamma_{i, j, L}}$.

Lemma 2.5. Under the hypotheses of Theorem 2.1 and assuming that the norm $\|N\|_{C^{\Sigma_{i, j}}}$ is sufficiently small in the ball of radius 3 , we have that $\mathcal{T}: \Gamma_{i, j-1, L} \longrightarrow$ $\Gamma_{i, j-1, L}$ sends the closed unit ball $\bar{B}_{1}^{j-1}$ of $\Gamma_{i, j-1, L}$ into itself, and it is a contraction there. 
Proof. As pointed out before, we can assume by scaling that the polynomial $K \leq$ is arbitrarily close to the identity in the unit ball of $X_{1}$, and that the polynomial $R$ is arbitrarily close to $A_{1, \lambda}$, uniformly in $\lambda \in \Lambda$.

As we did in the proof of Lemma 3.4 in [CFdIL00], we first show that $\mathcal{T}$ maps the unit ball of $\Gamma_{i, j-1, L}$ into itself. Recall that the operators $\mathcal{T}$ and $\tilde{H}$ were defined in (11) and (12), respectively. Let $K^{>} \in \bar{B}_{1}^{j-1}$. We claim that $\|\tilde{H}\|_{\Gamma_{i, j-1, L}}$ can be made arbitrarily small, by taking $\|N\|_{C^{\Sigma_{i, j}}}$ sufficiently small (which can be obtained by scaling). As a consequence, using also Lemma 2.4 and $\mathcal{T}=\mathcal{S}^{-1} \tilde{H}$, we deduce that $\left\|\mathcal{T}\left(K^{>}\right)\right\|_{\Gamma_{i, j-1, L}} \leq 1$.

To prove the claim, we already know that for every $\lambda \in \Lambda, D_{x}^{b} \tilde{H}_{\lambda}(0)=0$ for $0 \leq b \leq L$, and hence $D_{\lambda}^{a} D_{x}^{b} \tilde{H}_{\lambda}(0)=0$ for $0 \leq a \leq i, 0 \leq b \leq L$. Next we bound $D_{\lambda}^{a} D_{x}^{b} \tilde{H}_{\lambda}(x)$ for $(a, b) \in \Sigma_{i, j}$.

In the case $b \leq L$, by Taylor's theorem we have

$$
\left|D_{\lambda}^{a} D_{x}^{b} \tilde{H}_{\lambda}(x)\right| \leq C\left\|D_{\lambda}^{a} D_{x}^{L} \tilde{H}\right\|_{C^{0}}|x|^{L-b} .
$$

We decompose $\tilde{H}_{\lambda}$ as $\tilde{H}_{\lambda}=\tilde{H}_{\lambda}^{1}+\tilde{H}_{\lambda}^{2}$ with

$$
\tilde{H}_{\lambda}^{1}=-N_{\lambda} \circ K_{\lambda}^{\leq}-A_{\lambda} K_{\lambda}^{\leq}+K_{\lambda}^{\leq} \circ R_{\lambda}
$$

and

$$
\tilde{H}_{\lambda}^{2}=-N_{\lambda} \circ\left(K_{\lambda}^{\leq}+K_{\lambda}^{>}\right)+N_{\lambda} \circ K_{\lambda}^{\leq} .
$$

Using the previous expressions and that $R$ is a polynomial of degree $L$ in $x$, we have

$$
\left|D_{\lambda}^{a} D_{x}^{L} \tilde{H}_{\lambda}^{1}(x)\right| \leq\left\|D_{\lambda}^{a} D_{x}^{L+1}\left[-N_{\lambda} \circ K_{\lambda}^{\leq}+\left(K_{\lambda}^{\leq}-\mathrm{Id}\right) \circ R_{\lambda}\right]\right\|_{C^{0}}|x|
$$

and

$$
\begin{aligned}
D_{\lambda}^{a} D_{x}^{L} \tilde{H}_{\lambda}^{2} & =-\int_{0}^{1} \frac{d}{d s}\left\{D_{\lambda}^{a} D_{x}^{L}\left[N_{\lambda} \circ\left(K_{\lambda}^{\leq}+s K_{\lambda}^{>}\right)\right]\right\} d s \\
& \left.=-\int_{0}^{1} D_{\lambda}^{a} D_{x}^{L}\left[D_{x} N_{\lambda} \circ\left(K_{\lambda}^{\leq}+s K_{\lambda}^{>}\right) K_{\lambda}^{>}\right)\right] d s .
\end{aligned}
$$

In the case $b>L$, we write

$$
D_{\lambda}^{a} D_{x}^{b} \tilde{H}_{\lambda}=D_{\lambda}^{a} D_{x}^{b}\left[-N_{\lambda} \circ\left(K_{\lambda}^{\leq}+K_{\lambda}^{>}\right)+\left(K_{\lambda}^{\leq}-\mathrm{Id}\right) \circ R_{\lambda}\right] .
$$

The smallness assumptions on $N$ and $K \leq-$ Id together with the fact that $K^{>} \in$ $\Gamma_{i, j-1, L}$ give that (28), (29) and (30) are small and hence $\left|D_{\lambda}^{a} D_{x}^{b} \tilde{H}_{\lambda}(x)\right|$ and $\sup _{(\lambda, x)}\left|D_{\lambda}^{a} D_{x}^{L} \tilde{H}_{\lambda}(x)\right| /|x|$ are also small.

To compute the Lipschitz constant of $\mathcal{T}$, we write

$$
\begin{aligned}
\left(\mathcal{T}\left(K^{>}+\Delta\right)\right. & \left.-\mathcal{T}\left(K^{>}\right)\right)_{\lambda} \\
& =-\int_{0}^{1} \mathcal{S}^{-1} D_{x} N_{\lambda} \circ\left(K_{\lambda}^{\leq}+K_{\lambda}^{>}+s \Delta_{\lambda}\right) \Delta_{\lambda} d s .
\end{aligned}
$$


Taking the $D_{\lambda}^{a} D_{x}^{b}$ derivative of the previous expression with indices $(a, b)$ in $\Sigma_{i, j-1}$, we easily see that

$$
\left\|D_{\lambda}^{a} D_{x}^{b}\left[\mathcal{T}\left(K^{>}+\Delta\right)-\mathcal{T}\left(K^{>}\right)\right]\right\|_{C^{0}} \leq C\left\|\mathcal{S}^{-1}\right\|\left\|D_{x} N\right\|_{C^{\Sigma_{i, j-1}}}\|\Delta\|_{C^{\Sigma_{i, j-1}}} .
$$

When $b=L$, note that the $D_{\lambda}^{a} D_{x}^{b}$ derivative of $D N_{\lambda} \circ\left(K_{\lambda}^{\leq}+K_{\lambda}^{>}+s \Delta_{\lambda}\right) \Delta_{\lambda}$ is a sum of terms, each one having a derivative of $\Delta$ with respect to $x$ of order $\beta \leq L$, which is bounded by $C|x|^{L-\beta+1}$. Then the supremum of the $D_{\lambda}^{a} D_{x}^{b}$ derivative divided by $|x|$ is less than $C\left\|D_{x} N\right\|_{C^{\Sigma_{i, j-1}}}$.

We conclude that, by the smallness requirement on $N$, the map $\mathcal{T}$ can be made a contraction in $\bar{B}_{1}^{j-1}$.

As a consequence of Lemma 2.5, $\mathcal{T}$ has a unique fixed point $K^{>}$in the unit ball of $\Gamma_{i, j-1, L}$. Since, for every $\lambda \in \Lambda$ fixed, the $K_{\lambda}^{>}$obtained in Lemma 2.5 belongs to the closed unit ball of $\Gamma_{i+j-1, L}$, by uniqueness it must coincide with the $K_{\lambda}^{>}$obtained in [CFAIINO].

2.2. Sharp regularity. Here we improve the differentiability conclusions of the previous section from $K^{>} \in C^{\Sigma_{i, j-1}}$ to $K^{>} \in C^{\Sigma_{i, j}}$.

We follow essentially the same arguments of Section 3 in [CFAll00], but we pay attention to the dependence on parameters. We have that $D_{x} K^{>}$satisfies the following equation, which is the parameter version of the analogous equation for $D K^{>}$in [CFAILOO)]:

$$
\begin{aligned}
A_{\lambda} D_{x} K_{\lambda}^{>} & -D_{x} K_{\lambda}^{>} \circ R_{\lambda} D_{x} R_{\lambda} \\
= & -D_{x} N_{\lambda} \circ\left(K_{\lambda}^{\leq}+K_{\lambda}^{>}\right)\left(D_{x} K_{\lambda}^{\leq}+D_{x} K_{\lambda}^{>}\right) \\
& -A_{\lambda} D_{x} K_{\lambda}^{\leq}+D_{x} K_{\lambda}^{\leq} \circ R_{\lambda} D_{x} R_{\lambda} .
\end{aligned}
$$

This equation can be rewritten as

$$
\tilde{\mathcal{S}} D_{x} K_{\lambda}^{>}=\tilde{\mathcal{T}} D_{x} K_{\lambda}^{>}+U_{\lambda},
$$

where

$$
\begin{aligned}
(\tilde{\mathcal{S}} G)_{\lambda} & =A_{\lambda} G_{\lambda}-G_{\lambda} \circ R_{\lambda} D_{x} R_{\lambda}, \\
(\tilde{\mathcal{T}} G)_{\lambda} & =-D_{x} N_{\lambda} \circ\left(K_{\lambda}^{\leq}+K_{\lambda}^{>}\right) G_{\lambda},
\end{aligned}
$$

and

$$
U_{\lambda}=-D_{x} N_{\lambda} \circ\left(K_{\lambda}^{\leq}+K_{\lambda}^{>}\right) D_{x} K_{\lambda}^{\leq}-A_{\lambda} D_{x} K_{\lambda}^{\leq}+D_{x} K_{\lambda}^{\leq} \circ R_{\lambda} D_{x} R_{\lambda}
$$

with $K^{>}$being the function obtained in Lemma 2.5, which belongs to $\Gamma_{i, j-1, L}$. We consider $\tilde{\mathcal{S}}$ and $\tilde{\mathcal{T}}$ acting on the space $\Gamma_{i, s, L-1}$ defined in (17) with $Y=$ $\mathcal{L}\left(X_{1}, X\right)$.

Lemma 2.6. Under the assumptions of Theorem 2.1 and under the smallness assumptions arranged by scaling, if $s \in \mathbb{N}$ and $L-1 \leq s \leq j-1$ then $\tilde{\mathcal{S}}$ and $\tilde{\mathcal{T}}$ are bounded linear operators from $\Gamma_{i, s, L-1}$ into itself. Moreover, taking $\|N\|_{C^{\Sigma_{i, j}}}$ sufficiently small, $\tilde{\mathcal{S}}$ is invertible and $\left\|\tilde{\mathcal{S}}^{-1}\right\|\|\tilde{\mathcal{T}}\|<1$. 
Proof. We start studying the operator $\tilde{\mathcal{T}}$. Let $G \in \Gamma_{i, s, L-1}$ and $(a, b) \in \Sigma_{i, s}$. Using the Faa di Bruno's formula, the same arguments in the proof of Lemma 2.3, we have that

$$
\begin{aligned}
D_{\lambda}^{a} D_{x}^{b}\left[D_{x} N_{\lambda} \circ\left(K_{\lambda}^{\leq}+K_{\lambda}^{>}\right) G_{\lambda}\right] & \\
& =\sum_{\substack{0 \leq l \leq a, 0 \leq m \leq b}} C D_{\lambda}^{l} D_{x}^{m}\left[D_{x} N_{\lambda} \circ\left(K_{\lambda}^{\leq}+K_{\lambda}^{>}\right)\right] D_{\lambda}^{a-l} D_{x}^{b-m} G_{\lambda}
\end{aligned}
$$

and

$$
\begin{aligned}
D_{\lambda}^{l} D_{x}^{m}\left[D_{x} N_{\lambda} \circ\left(K_{\lambda}^{\leq}+K_{\lambda}^{>}\right)\right] & \sum_{(\alpha, \beta) \in \Sigma_{l, m}^{*}} \sum_{\substack{i_{1}+\cdots+i_{\beta}=l-\alpha, j_{1}+\cdots+j_{\beta}=m}} C D_{\lambda}^{\alpha} D_{x}^{\beta} D_{x} N_{\lambda} \circ\left(K_{\lambda}^{\leq}+K_{\lambda}^{>}\right) \\
& \cdot D_{\lambda}^{i_{1}} D_{x}^{j_{1}}\left(K_{\lambda}^{\leq}+K_{\lambda}^{>}\right) \cdots \quad D_{\lambda}^{i_{\beta}} D_{x}^{j_{\beta}}\left(K_{\lambda}^{\leq}+K_{\lambda}^{>}\right),
\end{aligned}
$$

where $C$ denotes combinatorial constants which are different in each term. Therefore, each term in (33) has a derivative $D_{\lambda}^{\alpha} D_{x}^{\gamma} N$ with $(\alpha, \gamma) \in \Sigma_{a, b+1} \subset \Sigma_{i, s+1} \subset$ $\Sigma_{i, j}$, and a derivative $D_{\lambda}^{\alpha} D_{x}^{\beta} G$ with $0 \leq \alpha \leq a, 0 \leq \beta \leq b$.

Note that $\left|D_{\lambda}^{\alpha} D_{x}^{\beta} G_{\lambda}(x)\right| \leq \frac{1}{(L-\beta)_{+} !}\|G\|_{\Gamma_{i, s, L-1}}|x|^{(L-\beta)_{+}}$. Therefore,

$$
\mid\left[\left.D_{\lambda}^{a} D_{x}^{b}\left[D_{x} N_{\lambda} \circ\left(K_{\lambda}^{\leq}+K_{\lambda}^{>}\right) G_{\lambda}\right](x)\left|\leq C\|N\|_{C^{\Sigma_{i, j}}}\|G\|_{\Gamma_{i, s, L-1}}\right| x\right|^{(L-b+m)_{+}},\right.
$$

and in particular

$$
\left\|D_{\lambda}^{a} D_{x}^{L-1}\left[D_{x} N_{\lambda} \circ\left(K_{\lambda}^{\leq}+K_{\lambda}^{>}\right) G_{\lambda}\right]\right\|_{\Gamma_{i, s, L-1}} \leq C\|N\|_{C^{\Sigma_{i, j}}}\|G\|_{\Gamma_{i, s, L-1}}|x| .
$$

We conclude that $\tilde{\mathcal{T}}$ is bounded and $\|\tilde{\mathcal{T}}\|$ is as small as we need.

Next we check that $\left\|\tilde{\mathcal{S}}^{-1}\right\|$ is bounded. It is clear that the series

$$
\sum_{k=0}^{\infty} A_{\lambda}^{-(k+1)} \eta_{\lambda} \circ R_{\lambda}^{k} D_{x} R_{\lambda}^{k}
$$

provides the formal solution of $\tilde{\mathcal{S}} H=\eta$. We will show that

$$
\sum_{k=0}^{\infty}\left\|A_{\lambda}^{-(k+1)} \eta_{\lambda} \circ R_{\lambda}^{k} D_{x} R_{\lambda}^{k}\right\|_{\Gamma_{i, s, L-1}} \leq C\|\eta\|_{\Gamma_{i, s, L-1}}
$$

for some constant $C$.

For $(a, b) \in \Sigma_{i, s}$, we have

$$
\begin{aligned}
D_{\lambda}^{a} D_{x}^{b}\left[A_{\lambda}^{-(k+1)} \eta_{\lambda} \circ R_{\lambda}^{k} D_{x} R_{\lambda}^{k}\right] & \\
& =\sum_{m=0}^{a} C\left[D_{\lambda}^{a-m} A_{\lambda}^{-(k+1)}\right] D_{\lambda}^{m} D_{x}^{b}\left[\eta_{\lambda} \circ R_{\lambda}^{k} D_{x} R_{\lambda}^{k}\right] .
\end{aligned}
$$

Using the Faa-di-Bruno formula, (25), (26) and

$$
\left|D_{\lambda}^{\alpha} D_{x}^{\beta} \eta_{\lambda}\left(R_{\lambda}^{k}(x)\right)\right| \leq C\|\eta\|_{\Gamma_{i, s, L-1}}\left|R_{\lambda}^{k}(x)\right|^{(L-\beta)_{+}},
$$


we deduce that the sum in (35) is bounded by

$$
C\|\eta\|_{\Gamma_{i, s, L-1}}(k+1)^{i}\left(\left\|A_{\lambda}^{-1}\right\|\left(\left\|A_{1, \lambda}\right\|+\varepsilon\right)^{L+1}\right)^{k} .
$$

When $b=L-1$, if $\beta \leq L-1$ then $\left|R_{\lambda}^{k}(x)\right|^{(L-\beta)_{+}}$provides a factor $|x|$. Otherwise $\beta>L-1$ and then, since $j_{1}+\cdots+j_{\beta}=b=L-1$ in the Faa di Bruno formula, at least one index $j$ of $\left\{j_{1}, \ldots, j_{\beta}\right\}$ must be zero and then $D_{\lambda}^{i_{1}} D_{x}^{j_{1}} R_{\lambda}^{k} \cdots D_{\lambda}^{i_{\beta}} D_{x}^{j_{\beta}} R_{\lambda}^{k}$ provides a factor $|x|$. We conclude that

$$
\begin{aligned}
&\left|D_{\lambda}^{a} D_{x}^{L-1}\left[A_{\lambda}^{-(k+1)} \eta \circ R_{\lambda}^{k} D R_{\lambda}^{k}\right](x)\right| /|x| \\
& \leq C\|\eta\|_{\Gamma_{i, s, L-1}}(k+1)^{i}\left(\left\|A_{\lambda}^{-1}\right\|\left(\left\|A_{1, \lambda}\right\|+\varepsilon\right)^{L+1}\right)^{k} .
\end{aligned}
$$

The proof of Lemma 2.6 is now complete.

To finish the proof of Theorem 2.1, that is, to prove that $K^{>} \in C^{\Sigma_{i, j}}$, we use Lemma 2.6 exactly in the same way as we used Lemma 3.6 in [CF'dLO0] to prove of Proposition 3.5 in [CFतlLON].

\section{Proof of Theorem [2}

The following is the main result of this section. It is a sharp regularity result, in the sense that we assume $F \in C^{\Sigma_{i, j}}$ and we obtain a manifold in the same class $C^{\Sigma_{i, j}}$. Here we do not assume $F$ to be jointly $C^{r}$.

Theorem 3.1. Let $F: \Lambda \times U \longrightarrow X$ be a map such that:

1) For some $(i, j) \in\left(\mathbb{Z}^{+}\right)^{2}$,

$$
F \in C^{\Sigma_{i, j}}
$$

where $\Sigma_{i, j}$ is the set introduced in Definition 1.5, and $C^{\Sigma_{i, j}}$ denotes the set of functions which have all the derivatives of indices in $\Sigma_{i, j}$ defined, continuous and bounded.

2) Conditions 0)-4) of Theorem 1.8 are satisfied.

3) $L+1 \leq j$.

Then, we can find maps $K \in C^{\Sigma_{i, j}}$ and $R \in C^{\Sigma_{i, j}}$ satisfying all the properties stated in Theorem [.?.

We recall that in the previous theorem -and throughout this section- we write the invariant manifold as a graph, and hence the parameterization $K$ is of the form

$$
K=\left(K^{1}, K^{2}\right)=(\mathrm{Id}, w)
$$

where, to simplify notation, we have renamed by $w$ the second component $K^{2}$ of $K$. It follows that

$$
R=F^{1} \circ K .
$$

The proof of Theorem 1.2 using Theorem 3.1 is immediate. It uses (36) together with the same argument in the proof of Theorem [1.1 using Theorem 2.1 (see the previous section). 
The next lemma corresponds to Lemma 2.2. It follows from the arguments explained before the statement of Lemma 2.2.

Lemma 3.2. Assume that $F \in C^{\Sigma_{i, j}}$ for some $j \geq L$, and that hypotheses 0 ), 1), 3) and 4) of Theorem 1.9 are satisfied uniformly in $\lambda$. Then, for every $\lambda$ there exists a unique polynomial $w_{\lambda}^{\leq}$of degree $L$ such that

$$
\begin{aligned}
& w_{\lambda}^{\leq}(x)=\mathcal{N}\left(w_{\lambda}^{\leq}\right)(x)+o\left(|x|^{L}\right), \\
& w_{\lambda}^{\leq}(0)=0, \quad D_{x} w_{\lambda}^{\leq}(0)=0 .
\end{aligned}
$$

Moreover, $w \leq C^{i, \infty}$ and, with a suitable scaling of $F_{\lambda}$, we can get $w_{\lambda}^{\leq}$as small as needed.

Regarding the operator $\mathcal{N}$ in (37), we recall (see [CFdIL00]) that, in the present setting, the functional equation $F \circ K=K \circ R$ has become $w=\mathcal{N}(w)$, where

$$
(\mathcal{N}(w))_{\lambda}=A_{2, \lambda}^{-1} w_{\lambda} \circ \psi_{w_{\lambda}}-A_{2, \lambda}^{-1} N_{2, \lambda} \circ\left(\mathrm{Id}, w_{\lambda}\right)
$$

and

$$
\psi_{w_{\lambda}}=A_{1, \lambda}+N_{1, \lambda} \circ\left(\mathrm{Id}, w_{\lambda}\right)+B_{\lambda} w_{\lambda}
$$

we will write $\psi_{\lambda}$ for $\psi_{w_{\lambda}}$, to simplify notation. We also write $w_{\lambda}=w_{\lambda}^{\leq}+w_{\lambda}^{>}$, where $w_{\lambda}^{\leq}$is the polynomial in $x$ given by Lemma 3.2, and then equation (39) becomes

$$
w^{>}=\mathcal{M}\left(w^{>}\right),
$$

where, consistent with our notation of denoting the parameter as a subindex, we will write

$$
\left(\mathcal{M}\left(w^{>}\right)\right)_{\lambda}=\left(\mathcal{N}\left(w^{\leq}+w^{>}\right)\right)_{\lambda}-w_{\lambda}^{\leq}=\mathcal{N}_{\lambda}\left(w_{\lambda}^{\leq}+w_{\lambda}^{>}\right)-w_{\lambda}^{\leq} .
$$

The starting point of the proof of Theorem 3.1 is the following result from the previous paper [CFdIL00]. Under the hypotheses of Theorem 3.1, we have that for every $\lambda, F_{\lambda}$ is a $C^{i+j}$ function of $x$. Hence, from the results of [CF'ILO0], we know that the sequence of iterates

$$
w_{n}^{>}=\mathcal{M}^{n}(0)
$$

(which starts with $w_{0}^{>}=0$ ) converges uniformly in $B_{1}$, together with all its derivatives in $x$ up to order $i+j-1$, to the solution $w_{\infty}^{>}$produced in that paper. In particular, since all estimates are uniform in $\lambda$, we have that $w_{n}^{>}$and $D_{x} w_{n}^{>}$ converge uniformly in $\Lambda \times B_{1}$ to $w_{\infty}^{>}$and $D_{x} w_{\infty}^{>}$, respectively.

The proof of Theorem 3.1 proceeds by expressing, for every function $w^{>}$, the derivative $D_{\lambda}^{i} D_{x}^{j} \mathcal{M}\left(w^{>}\right)$as

$$
D_{\lambda}^{i} D_{x}^{j} \mathcal{M}\left(w^{>}\right)=\mathcal{A}\left(w^{>}\right) D_{\lambda}^{i} D_{x}^{j} w^{>}+\mathcal{B}\left(w^{>}\right),
$$

where $\mathcal{A}\left(w^{>}\right)$is a linear operator, and $\mathcal{B}\left(w^{>}\right)$is an expression that involves only derivatives of lower order. We will show that $\mathcal{A}_{n}:=\mathcal{A}\left(w_{n}^{>}\right)$is a contractive operator. From this it will follow that, when $D_{\lambda}^{k} D_{x}^{l} \mathcal{M}^{n}(0)$ converge uniformly on compact sets as $n \rightarrow \infty$ for all $(k, l)$ of lower order than $(i, j)$, then $D_{\lambda}^{i} D_{x}^{j} \mathcal{M}^{n}(0)$ also converges uniformly on compact sets. 
From the finite increment formula for the derivatives of the form $D_{\lambda}^{i-1} D_{x}^{j} w_{n}^{>}$ or $D_{\lambda}^{i} D_{x}^{j-1} w_{n}^{>}$, we will recover the finite increment formula for their limits as $n \rightarrow \infty$. This will give us that the limit of $D_{\lambda}^{i} D_{x}^{j} w_{n}^{>}$as $n$ tends to infinity is indeed a true derivative.

We will encounter the difficulty that in infinite dimensions, continuity on compact sets does not imply continuity and boundedness on a ball, and we will need to use an extra argument. We will show that the derivative which we have obtained as a limit satisfies a certain functional equation. By studying this equation, it will be possible to show that, when the derivatives of lower order are continuous and bounded, then the limit of $D_{\lambda}^{i} D_{x}^{j} \mathcal{M}^{n}(0)$ is also continuous and bounded.

Next we start with the precise results that will lead to Theorem 3.1. Through this section, we use the notation $D_{1}=D_{x_{1}}$ and $D_{2}=D_{x_{2}}$ for derivatives with respect to the variables in $X_{1}$ and in $X_{2}$, respectively.

Proposition 3.3. Assume that $F$ and $w^{>}$belong to $C^{\Sigma_{i, j}}$. Then, the following formula holds:

$$
\begin{aligned}
& {\left[D_{\lambda}^{i} D_{x}^{j} \mathcal{M}\left(w^{>}\right)\right]_{\lambda}} \\
& \quad=\quad A_{2, \lambda}^{-1} D_{\lambda}^{i} D_{x}^{j} w_{\lambda}^{>} \circ \psi_{\lambda}\left(D_{x} \psi_{\lambda}\right)^{\otimes j} \\
& \quad+A_{2, \lambda}^{-1}\left[D_{x} w_{\lambda} \circ \psi_{\lambda}\left(D_{2} N_{1, \lambda} \circ\left(\mathrm{Id}, w_{\lambda}\right)+B_{\lambda}\right)-D_{2} N_{2, \lambda} \circ\left(\mathrm{Id}, w_{\lambda}\right)\right] D_{\lambda}^{i} D_{x}^{j} w_{\lambda}^{>} \\
& \quad+V_{i, j}\left(w_{\lambda}^{>}\right)
\end{aligned}
$$

where $V_{i, j}$ is a multilinear operator in $D_{\lambda}^{a} D_{x}^{b} w^{>}$with $(a, b) \in \Sigma_{i, j}-\{(i, j)\}$, whose coefficients are derivatives of $N$ of orders in $\Sigma_{i, j}$, or derivatives of $A_{1, \lambda}$ or $B_{\lambda}$ with respect to $\lambda$ of orders in $\{0, \ldots, i\}$.

This proposition is an immediate consequence of the following three lemmas, which provide a quite detailed structure of the derivatives of the composition.

As in the previous section, here we see that the rules for computing higher order derivatives with respect to parameters of a composition suggest the introduction of special sets of indices.

We introduce two more auxiliary sets of indices for $i \in \mathbb{N}, j \geq 1$ :

$$
\begin{aligned}
& \Sigma_{i, 0}^{* *}=\left\{(a, b, 0) \in\left(\mathbb{Z}^{+}\right)^{3} \mid a+b \leq i, b \geq 1\right\} \cup\{(i, 0,0)\} \\
& \Sigma_{i, j}^{* *}=\left\{(a, b, c) \in\left(\mathbb{Z}^{+}\right)^{3} \mid a+b+c \leq i+j, a \leq i, c \leq j, b \geq 1\right\} .
\end{aligned}
$$

In the rest of the section, $C$ means a generic constant which depends only on the map $F$, and which is independent of the functions $w$ and $w^{>}$. Hence, the same letter may denote different constants in different places.

Lemma 3.4. Under the hypotheses of Proposition 3.3, we have that $\mathcal{M}\left(w^{>}\right) \in$ $C^{\Sigma_{i, j}}$ and that

$$
D_{\lambda}^{i} D_{x}^{j}\left[\mathcal{M}\left(w^{>}\right)\right]
$$


is an expression of the form

$$
\begin{aligned}
& A_{2, \lambda}^{-1} \sum_{(a, b) \in \Sigma_{i, j}^{*}} \sum_{\substack{i_{1}+\ldots+i_{b}=i-a, j_{1}+\cdots+j_{b}=j}} C D_{\lambda}^{a} D_{x}^{b} w_{\lambda} \circ \psi_{\lambda} D_{\lambda}^{i_{1}} D_{x}^{j_{1}} \psi_{\lambda} \cdots D_{\lambda}^{i_{b}} D_{x}^{j_{b}} \psi_{\lambda} \\
& +C\left(D_{\lambda} A_{2, \lambda}^{-1}\right) D_{\lambda}^{i-1} D_{x}^{j}\left[w_{\lambda} \circ \psi_{\lambda}\right]+\cdots+C\left(D_{\lambda}^{i} A_{2, \lambda}^{-1}\right) D_{x}^{j}\left[w_{\lambda} \circ \psi_{\lambda}\right] \\
& -A_{2, \lambda}^{-1} \sum_{(a, b, c) \in \Sigma_{i, j}^{* *}} \sum_{\substack{i_{1}+\cdots+i_{b}=i-a, j_{1}+\cdots+j_{b}=j-c}} C D_{\lambda}^{a} D_{1}^{c} D_{2}^{b} N_{2, \lambda} \circ\left(\mathrm{Id}, w_{\lambda}\right) \\
& \cdot D_{\lambda}^{i_{1}} D_{x}^{j_{1}} w_{\lambda} \cdots D_{\lambda}^{i_{b}} D_{x}^{j_{b}} w_{\lambda} \\
& -C\left(D_{\lambda} A_{2, \lambda}^{-1}\right) D_{\lambda}^{i-1} D_{x}^{j}\left[N_{2, \lambda} \circ\left(\operatorname{Id}, w_{\lambda}\right)\right]-\cdots-C\left(D_{\lambda}^{i} A_{2, \lambda}^{-1}\right) D_{x}^{j}\left[N_{2, \lambda} \circ\left(\mathrm{Id}, w_{\lambda}\right)\right] \\
& -D_{\lambda}^{i} D_{x}^{j} w_{\lambda}^{\leq} \text {. }
\end{aligned}
$$

This lemma follows directly from the next two lemmas.

Lemma 3.5. Let $w \in C^{\Sigma_{i, j}}$ and $\psi \in C^{i, j}$. Then the derivatives of $w_{\lambda} \circ \psi_{\lambda}(x)=$ $w(\lambda, \psi(\lambda, x))$ have the form

$$
\begin{aligned}
& D_{\lambda}^{i} D_{x}^{j}\left[w_{\lambda} \circ \psi_{\lambda}\right] \\
& =\sum_{(a, b) \in \Sigma_{i, j}^{*},} \sum_{\substack{i_{1}+\cdots+i_{b}=-a, j_{1}+\cdots+j_{b}=j}} C D_{\lambda}^{a} D_{x}^{b} w_{\lambda} \circ \psi_{\lambda} \cdot D_{\lambda}^{i_{1}} D_{x}^{j_{1}} \psi_{\lambda} \cdots D_{\lambda}^{i_{b}} D_{x}^{j_{b}} \psi_{\lambda},
\end{aligned}
$$

where $C$ is a combinatorial coefficient which depends on $a, b, i_{1}, \ldots, i_{b}, j_{1}, \ldots, j_{b}$, and $\Sigma_{i, j}^{*}$ is defined by (18).

Proof. The proof of this lemma is contained in the proof of Lemma 2.3 when one identifies $w$ and $\psi$ with $H$ and $R^{k}$ of that lemma, respectively.

Lemma 3.6. Let $N \in C^{\Sigma_{i_{0}, j_{0}}}$ and $w \in C^{\Sigma_{i_{0}, j_{0}}}$. Then, we have that $N_{\lambda}$ 。 $\left(\mathrm{Id}, w_{\lambda}\right) \in C^{\Sigma_{i_{0}, j_{0}}}$, and for $(i, j) \in \Sigma_{i_{0}, j_{0}}$

$$
\begin{aligned}
& D_{\lambda}^{i} D_{x}^{j}\left[N_{\lambda} \circ\left(\mathrm{Id}, w_{\lambda}\right)\right] \\
& \quad=\sum_{(a, b, c) \in \Sigma_{i, j}^{* *} i_{1}, j_{1}, \ldots, i_{b}, j_{b}} C D_{\lambda}^{a} D_{1}^{c} D_{2}^{b} N_{\lambda} \circ\left(\mathrm{Id}, w_{\lambda}\right) D_{\lambda}^{i_{1}} D_{x}^{j_{1}} w_{\lambda} \cdots D_{\lambda}^{i_{b}} D_{x}^{j_{b}} w_{\lambda}
\end{aligned}
$$

where $i_{1}+\cdots+i_{b}=i-a, j_{1}+\cdots+j_{b}=j-c$, and $C$ is a coefficient depending on the indices. The only term in the sum (42) that contains $D_{\lambda}^{i} D_{x}^{j} w_{\lambda}$ is $D_{2} N_{\lambda}$ 。 $\left(\mathrm{Id}, w_{\lambda}\right) D_{\lambda}^{i} D_{x}^{j} w_{\lambda}$.

Proof. We begin by computing the $i^{\text {th }}$ derivative of $N_{\lambda} \circ\left(\mathrm{Id}, w_{\lambda}\right)$ with respect to $\lambda$, which we denote by $T_{i}=D_{\lambda}^{i}\left[N_{\lambda} \circ\left(\mathrm{Id}, w_{\lambda}\right)\right]$. We have

$$
D_{\lambda}^{i}\left[N_{\lambda} \circ\left(\mathrm{Id}, w_{\lambda}\right)\right]=\sum_{(a, b) \in \Sigma_{i, 0}^{*}} \sum C D_{\lambda}^{a} D_{2}^{b} N_{\lambda} \circ\left(\mathrm{Id}, w_{\lambda}\right) D_{\lambda}^{i_{1}} w_{\lambda} \cdots D_{\lambda}^{i_{b}} w_{\lambda},
$$


where the second sum is taken for $i_{1}+\cdots+i_{b}=i-a$, and $C$ is a coefficient which depends on $a, b, i_{1}, \ldots, i_{b}$. This follows from Lemma 3.5 identifying $N_{\lambda}(x,$.$) with$ $w_{\lambda}$, and $w_{\lambda}$ with $\psi_{\lambda}$.

Note that $D_{x}^{j} T_{i}=D_{\lambda}^{i} D_{x}^{j}\left[N_{\lambda} \circ\left(\mathrm{Id}, w_{\lambda}\right)\right]$. The rest of the proof is by induction in $j$. When $j=0$, formula (42) holds. The induction step incrementing $j$ corresponds to taking one more derivative with respect to $x$. Assuming that (42) is true for $j$,

$$
\begin{aligned}
& D_{x}^{j+1} T_{i}= \sum_{(a, b, c) \in \Sigma_{i, j}^{* *}} \sum_{i_{1}, j_{1}, \ldots, i_{b}, j_{b}} C\left[D_{\lambda}^{a} D_{1}^{c+1} D_{2}^{b} N_{\lambda} \circ\left(\mathrm{Id}, w_{\lambda}\right)\right. \\
&\left.+D_{\lambda}^{a} D_{1}^{c} D_{2}^{b+1} N_{\lambda} \circ\left(\mathrm{Id}, w_{\lambda}\right) D_{x} w_{\lambda}\right] \cdot\left[D_{\lambda}^{i_{1}} D_{x}^{j_{1}} w_{\lambda} \cdots D_{\lambda}^{i_{b}} D_{x}^{j_{b}} w_{\lambda}\right] \\
&+\sum_{(a, b, c) \in \Sigma_{i, j}^{* *} i_{1}, j_{1}, \ldots, i_{b}, j_{b}} C D_{\lambda}^{a} D_{1}^{c} D_{2}^{b} N_{\lambda} \circ\left(\mathrm{Id}, w_{\lambda}\right) . \\
& D_{x}\left[D_{\lambda}^{i_{1}} D_{x}^{j_{1}} w_{\lambda} \cdots D_{\lambda}^{i_{b}} D_{x}^{j_{b}} w_{\lambda}\right] .
\end{aligned}
$$

From the previous formula we see that taking one more derivative has the effect that each term labeled with indices $(a, b, c)$ generates three new terms labeled with indices $(a, b, c),(a, b+1, c)$ and $(a, b, c+1)$, except for the term with $b=0$ which only appears when $j=0$ and it must have indices $(i, 0,0)$. Such term only generates two terms with indices $(i+1,0,0)$ and $(i, 1,0)$.

For $j>0$ we decompose $\Sigma_{i, j}^{* *}=\tilde{\Sigma}_{i, j}^{0} \cup \cdots \cup \tilde{\Sigma}_{i, j}^{m} \cup \cdots \cup \tilde{\Sigma}_{i, j}^{i}$, where

$$
\tilde{\Sigma}_{i, j}^{m}=\{(m, b, c) \mid b+c \leq i+j-m, c \leq j, b \geq 1\} .
$$

In this way, it is easy to see that the symbolic process

$$
(a, b, c) \mapsto(a, b, c)+(a, b+1, c)+(a, b, c+1)
$$

is exhaustive from $\tilde{\Sigma}_{i, j}^{m}$ to $\tilde{\Sigma}_{i, j+1}^{m}$. For the case $j=0$ the same argument works, but one has to consider the term $(i, 0,0)$ separately.

We note that the highest derivative of $w$, namely $D_{\lambda}^{i} D_{x}^{j} w$, appears multiplied by $D_{2} N$ (this means $a=c=0, b=1$ ).

As we already mentioned, Lemma 3.4 follows directly from Lemmas 3.5 and 3.6, while Proposition 3.3 is an immediate consequence of Lemmas 3.4, 3.5 and 3.6 .

Now we show inductively the existence of derivatives assuming that all the lower order ones are defined.

Given a subset $S \subset \Lambda \times B_{1}$, we introduce the space

$$
\begin{aligned}
& \Gamma_{i, j}^{*}(S) \\
& =\left\{\left.v \in C^{0}\left(S, L^{i}\left(\Lambda_{0} ; L^{j}\left(X_{1} ; X_{2}\right)\right)\right)\left|\sup _{(\lambda, x) \in S, x \neq 0}\right| v_{\lambda}(x)|/| x\right|^{(L-j+1)_{+}}<\infty\right\}
\end{aligned}
$$


with $\|v\|_{\Gamma_{i, j}^{*}(S)}=\sup _{(\lambda, x) \in S, x \neq 0}\left|v_{\lambda}(x)\right| /|x|^{(L-j+1)_{+}}$. Given a set of indices $\Sigma$, we also define the space

$\Gamma(\Sigma, S)$

$$
=\left\{\left.w \in C^{\Sigma}(S)\left|\sup _{(\lambda, x) \in S, x \neq 0}\right| D_{\lambda}^{a} D_{x}^{b} w_{\lambda}(x)|/| x\right|^{(L-b+1)_{+}}<\infty, \forall(a, b) \in \Sigma\right\}
$$

with $\|w\|_{\Gamma(\Sigma, S)}=\max _{(a, b) \in \Sigma} \sup _{(\lambda, x) \in S, x \neq 0}\left|D_{\lambda}^{a} D_{x}^{b} w_{\lambda}(x)\right| /|x|^{(L-b+1)_{+}}$.

Proposition 3.7. Assume that $F \in C^{\Sigma_{i_{0}, j_{0}}}\left(\Lambda \times B_{1}\right)$, with $j_{0} \geq L+1$, and that $N$ has small enough $C^{\Sigma_{i_{0}, j_{0}}}$ norm. Let $(i, j) \in \Sigma_{i_{0}, j_{0}}$, and consider

$$
\Sigma_{i, j}^{\prime}:=\Sigma_{i, j}-\{(i, j)\}
$$

Let $w_{n}^{>}$be the sequence of families of functions defined by

$$
w_{n}^{>}=\mathcal{M}^{n}(0) \text {. }
$$

Assume that:

1) $w_{\infty}^{>} \in C^{\Sigma_{i, j}^{\prime}}\left(\Lambda \times B_{1}\right)$.

2) For every compact set $G \subset \Lambda \times B_{1}, w_{n}^{>}$converges to $w_{\infty}^{>}$in $\Gamma\left(\Sigma_{i, j}^{\prime}, G\right)$. Then,

a) $w_{\infty}^{>} \in C^{\Sigma_{i, j}}\left(\Lambda \times B_{1}\right)$.

b) For every compact set $G \subset \Lambda \times B_{1}, D_{\lambda}^{i} D_{x}^{j} w_{n}^{>}$converges to $D_{\lambda}^{i} D_{\lambda}^{j} w_{\infty}^{>}$in $\Gamma_{i, j}^{*}(G)$, and therefore $w_{n}^{>}$converges to $w_{\infty}^{>}$in $\Gamma\left(\Sigma_{i, j}, G\right)$.

At the end of this section we will see that Theorem 3.1 follows easily from the previous proposition.

To prove Proposition 3.7, we first introduce some notation and we establish some preliminary lemmas.

First we remark that, since $j_{0} \geq 1$, if $F \in C^{\Sigma_{i_{0}, j_{0}}}$ then $N=F-D_{x} F(0) \in$ $C^{\Sigma_{i_{0}, j_{0}}}$. We write (43) in inductive form,

$$
w_{n+1}^{>}=\mathcal{M}\left(w_{n}^{>}\right), \quad n \in \mathbb{Z}^{+}, \quad w_{0}^{>}=0 .
$$

We introduce the notation

$$
w_{n}=w^{\leq}+w_{n}^{>}
$$

and

$$
\psi_{\lambda, n}(x)=A_{1, \lambda} x+N_{1, \lambda}\left(x, w_{\lambda, n}(x)\right)+B_{\lambda} w_{\lambda, n}(x), \quad n \in \mathbb{Z}^{+} \cup\{\infty\} .
$$

Differentiating in (44), we have

$$
D_{\lambda}^{i} D_{x}^{j} w_{\lambda, n+1}^{>}=D_{\lambda}^{i} D_{x}^{j}\left[\mathcal{M}\left(w_{\lambda, n}^{>}\right)\right] .
$$

By Proposition 3.3 and Lemmas 3.4 to 3.6, we can expand the right hand side of (46) as a sum of derivatives of different orders. We define a linear operator $\mathcal{A}_{n}$ in such a way that $\mathcal{A}_{n} D_{\lambda}^{i} D_{x}^{j} w_{n}^{>}$contains the terms in such expansion with $D_{\lambda}^{i} D_{x}^{j} w_{n}^{>}$. We take $\mathcal{B}_{n}$ as the sum of all the remaining terms of the expansion. We allow $n$ to be $\infty$, meaning the limit. 
More explicitly, for $n \in \mathbb{Z}^{+} \cup\{\infty\}$, we define

$$
\begin{aligned}
& {\left[\mathcal{A}_{n} v\right]_{\lambda}=A_{2, \lambda}^{-1} v_{\lambda} \circ \psi_{\lambda, n}\left(D_{x} \psi_{\lambda, n}\right)^{\otimes j}} \\
& \quad+A_{2, \lambda}^{-1}\left[D_{x} w_{\lambda, n} \circ \psi_{\lambda, n}\left(D_{2} N_{1, \lambda} \circ\left(\mathrm{Id}, w_{\lambda, n}\right)+B_{\lambda}\right)-D_{2} N_{2, \lambda} \circ\left(\mathrm{Id}, w_{\lambda, n}\right)\right] v_{\lambda}
\end{aligned}
$$

and

$$
\mathcal{B}_{n}=D_{\lambda}^{i} D_{x}^{j}\left[\mathcal{M}\left(w_{n}^{>}\right)\right]-\mathcal{A}_{n} D_{\lambda}^{i} D_{x}^{j} w_{n}^{>} .
$$

We have that $\mathcal{B}_{n}$ is the multilinear expression in the derivatives $D_{\lambda}^{a} D_{x}^{b} w_{n}^{>}$for $(a, b) \in \Sigma_{i, j}^{\prime},(a, b) \neq(0,0)$, which contains all terms in the expansion of the right hand side of (42) except for the two only ones which contain $D_{\lambda}^{i} D_{x}^{j} w_{n}^{>}$.

Note that $\mathcal{A}_{n}$ and $\mathcal{B}_{n}$ actually depend on $i$ and $j$, but for typographical reasons we suppress these indices from the notation.

To study the convergence of the sequence $D_{\lambda}^{i} D_{x}^{j} w_{n}^{>}$on compact sets, it will be useful to associate to each compact set $G \subset \Lambda \times B_{1}$, the larger compact set

$$
G^{*}=\bigcup_{n \in \mathbb{N} \cup\{\infty\}, l \in \mathbb{Z}^{+}}\left\{\left(\lambda, \psi_{\lambda, n}^{l}(x)\right) \mid(\lambda, x) \in G\right\} \subset \Lambda \times B_{1} .
$$

We have that $G^{*}$ is compact since $\psi_{\lambda, n} \rightarrow \psi_{\lambda, \infty}$ as $n \rightarrow \infty$, and since $\psi_{\lambda, n}$ are uniform contractions. The set $G^{*}$ has the important property of being invariant by $\left(\pi_{\lambda}, \psi_{\lambda, n}\right)$ for every $n \in \mathbb{N} \cup\{\infty\}$, where $\pi_{\lambda}(\lambda, x)=\lambda$.

Lemma 3.8. Assume the hypotheses of Proposition 3.7. Let $n \in \mathbb{Z}^{+} \cup\{\infty\}$. Then,

a) Given a compact set $G \subset \Lambda \times B_{1}, \mathcal{A}_{n}: \Gamma_{i, j}^{*}\left(G^{*}\right) \longrightarrow \Gamma_{i, j}^{*}\left(G^{*}\right)$ is a well defined linear bounded operator and, denoting by $Y=L\left(\Gamma_{i, j}^{*}\left(G^{*}\right), \Gamma_{i, j}^{*}\left(G^{*}\right)\right)$, its norm satisfies $\left\|\mathcal{A}_{n}\right\|_{Y} \leq \gamma<1$, for some constant $\gamma$ independent of $n$ and of the compact set $G$.

b) $\mathcal{B}_{n} \in \Gamma_{i, j}^{*}\left(G^{*}\right)$ and $\left\|\mathcal{B}_{n}\right\|_{\Gamma_{i, j}^{*}\left(G^{*}\right)} \leq M$ for some constant $M>0$ independent of $n$ (but possibly dependent on $G$ ).

c) For $n \in \mathbb{N}$ and $(\lambda, x) \in \Lambda \times B_{1},\left|D_{\lambda}^{i} D_{x}^{j} w_{\lambda, n}^{>}(x)\right| \leq M_{i, j}|x|^{(L-j+1)_{+}}$for some constant $M_{i, j}$ independent of $n$.

d) $\mathcal{B}_{n}$ converges uniformly on compact sets to $\mathcal{B}_{\infty}$.

Proof. As we mentioned in the beginning of this section, we already know that $w_{n}^{>}$converges to $w_{\infty}^{>}$in $\Gamma\left(\Sigma_{0,1}, \Lambda \times B_{1}\right)$. Using also hypotheses 1$)$ and 2) of Proposition 3.7 together with expressions (45) and (42), we deduce that, for $n \in \mathbb{Z}^{+} \cup\{\infty\}$ and $(\lambda, x) \in \Lambda \times B_{1}$,

$$
\begin{aligned}
\left|\psi_{\lambda, n}(x)\right| & \leq\left(\left\|A_{1, \lambda}\right\|+\varepsilon\right)|x|, \\
\left|D_{x} \psi_{\lambda, n}(x)\right| & \leq\left\|A_{1, \lambda}\right\|+\varepsilon,
\end{aligned}
$$

with $\varepsilon$ as small as needed. We also get that, for every compact subset of $\Lambda \times B_{1}$,

$$
\left|D_{\lambda}^{a} \psi_{\lambda, n}(x)\right| \leq C|x|, \quad a \leq i \text { if } j \geq 1, \text { or } a \leq i-1 \text { if } j=0,
$$

where $C$ is a constant independent of $n$ (but depending on the compact set). 
a) $\mathcal{A}_{n}$ is a linear operator. We estimate its norm:

$$
\begin{aligned}
& \left\|\mathcal{A}_{n} v\right\|_{\Gamma_{i, j}^{*}\left(G^{*}\right)} \\
& =\sup _{(\lambda, x) \in G^{*}} \frac{1}{|x|^{(L-j+1)_{+}}}\left\|A_{2, \lambda}^{-1}\right\|\left\{\left|v_{\lambda}\left(\psi_{\lambda, n}(x)\right)\right|\left|D_{x} \psi_{\lambda, n}(x)\right|^{j}\right. \\
& \quad+\left[\left|D_{x} w_{\lambda, n}\left(\psi_{\lambda, n}(x)\right)\right|\left|D_{2} N_{1, \lambda}\left(x, w_{\lambda, n}(x)\right)+B_{\lambda}\right|\right. \\
& \left.\left.\quad+\left|D_{2} N_{2, \lambda}\left(x, w_{\lambda, n}(x)\right)\right|\right]\left|v_{\lambda}(x)\right|\right\} \\
& \quad \sup _{(\lambda, x) \in G^{*}}\left\|A_{2, \lambda}^{-1}\right\|\left\{\|v\|_{\Gamma_{i, j}^{*}\left(G^{*}\right)} \frac{\left|\psi_{\lambda, n}(x)\right|^{(L-j+1)_{+}}}{|x|^{(L-j+1)_{+}}}\left|D_{x} \psi_{\lambda, n}(x)\right|^{j}\right. \\
& \quad+\left[\left|D_{x} w_{\lambda, n}\left(\psi_{\lambda, n}(x)\right)\right|\left|D_{2} N_{1, \lambda}\left(x, w_{\lambda, n}(x)\right)+B_{\lambda}\right|\right. \\
& \left.\left.\quad+\left|D_{2} N_{2, \lambda}\left(x, w_{\lambda, n}(x)\right)\right|\right]\|v\|_{\Gamma_{i, j}^{*}\left(G^{*}\right)}\right\} \\
& \leq\left\|A_{2, \lambda}^{-1}\right\|\left[\left(\left\|A_{1, \lambda}\right\|+\varepsilon\right)^{L+1}+\varepsilon\right]\|v\|_{\Gamma_{i, j}^{*}\left(G^{*}\right)}
\end{aligned}
$$

by (48), (49) and the fact that $\|N\|_{C^{1}}+\left\|B_{\lambda}\right\|$ is as small as we want.

b) By the previous lemmas, we know that if $F \in C^{\Sigma_{i_{0}, j_{0}}}$ then $N \in C^{\Sigma_{i_{0}, j_{0}}}$; if $w \in C^{\Sigma_{i, j}}$ then $N_{\lambda} \circ\left(\operatorname{Id}, w_{\lambda}\right) \in C^{\Sigma_{i, j}}$; and if $w^{>} \in C^{\Sigma_{i, j}}$ then $\mathcal{M}\left(w^{>}\right) \in C^{\Sigma_{i, j}}$.

From the way that $\mathcal{N}$ was constructed, we have

$$
\left[D_{x}^{\ell} \mathcal{M}(0)\right]_{\lambda}(0)=D_{x}^{\ell}\left[\left(\mathcal{N}(w \leq)_{\lambda}-w_{\lambda}^{\leq}\right](0)=0, \quad 0 \leq \ell \leq L .\right.
$$

Then

$$
\left[D_{\lambda}^{a} D_{x}^{b} \mathcal{M}(0)\right]_{\lambda}(0)=0, \quad 0 \leq a \leq i, \quad 0 \leq b \leq L,
$$

and therefore

$$
\mathcal{M}(0) \in \Gamma\left(\Sigma_{i, j}, \Lambda \times B_{1}\right) .
$$

To prove that $\left|\mathcal{B}_{n}(\lambda, x)\right| \leq M|x|^{(L-j+1)_{+}}$for $(\lambda, x) \in G^{*}$, we write $\mathcal{B}_{n}$ as:

$$
D_{\lambda}^{i} D_{x}^{j} \mathcal{M}(0)+D_{\lambda}^{i} D_{x}^{j}\left[\mathcal{M}\left(w_{n}^{>}\right)-\mathcal{M}(0)\right]-\mathcal{A}_{n} D_{\lambda}^{i} D_{x}^{j} w_{n}^{>} .
$$

Next, we study the terms in the formula for

$$
D_{\lambda}^{i} D_{x}^{j}\left[\mathcal{M}\left(w_{n}^{>}\right)-\mathcal{M}(0)\right]
$$

given by Lemma 3.4, and we see that all of them contain the factor $|x|^{(L-j+1)_{+}}$ and are bounded uniformly in $n$. Since $\mathcal{M}(0)=\mathcal{M}\left(w_{0}^{>}\right)$, we study the more general expression

$$
D_{\lambda}^{i} D_{x}^{j}\left[\mathcal{M}\left(w_{n}^{>}\right)-\mathcal{M}\left(w_{m}^{>}\right)\right]-\mathcal{A}_{n} D_{\lambda}^{i} D_{x}^{j} w_{n}^{>}+\mathcal{A}_{m} D_{\lambda}^{i} D_{x}^{j} w_{m}^{>},
$$

to be able to use the conclusions in the case $m=\infty$. 
Every term that appears in the decomposition of (52) given by Lemma 3.4 is the product of a bounded quantity times a factor of one of the following forms:

$$
\begin{aligned}
T_{1, \lambda}= & {\left[D_{\lambda}^{a} D_{1}^{c} D_{2}^{b} N_{2, \lambda} \circ\left(\mathrm{Id}, w_{\lambda, n}\right)\right.} \\
& \left.-D_{\lambda}^{a} D_{1}^{c} D_{2}^{b} N_{2, \lambda} \circ\left(\mathrm{Id}, w_{\lambda, m}\right)\right] D_{\lambda}^{i_{1}} D_{x}^{j_{1}} w_{\lambda, m} \cdots D_{\lambda}^{i_{b}} D_{x}^{j_{b}} w_{\lambda, m}
\end{aligned}
$$

with $(a, b, c) \in \Sigma_{i, j}^{* *}$ and $i_{1}+\cdots+i_{b}=i-k-a, 0 \leq k \leq i-a, j_{1}+\cdots+j_{b}=j-c$;

$$
\begin{gathered}
T_{2, \lambda}=D_{\lambda}^{a} D_{1}^{c} D_{2}^{b} N_{2, \lambda} \circ\left(\mathrm{Id}, w_{\lambda, n}\right)\left[D_{\lambda}^{i_{1}} D_{x}^{j_{1}} w_{\lambda, n} \cdots D_{\lambda}^{i_{b}} D_{x}^{j_{b}} w_{\lambda, n}\right. \\
\left.-D_{\lambda}^{i_{1}} D_{x}^{j_{1}} w_{\lambda, m} \cdots D_{\lambda}^{i_{b}} D_{x}^{j_{b}} w_{\lambda, m}\right]
\end{gathered}
$$

with $(a, b, c) \in \Sigma_{i, j}^{* *}$ and $i_{1}+\cdots+i_{b}=i-k-a, 0 \leq k \leq i-a, j_{1}+\cdots+j_{b}=j-c$;

$$
T_{3, \lambda}(x)=D_{\lambda}^{\tilde{z}} D_{x}^{\tilde{\jmath}} \psi_{\lambda, n}(x)-D_{\lambda}^{\tilde{\imath}} D_{x}^{\tilde{\jmath}} \psi_{\lambda, m}(x)
$$

with $\tilde{\imath} \leq i, \tilde{\jmath} \leq j,(\tilde{\imath}, \tilde{\jmath}) \neq(i, j)$;

$$
T_{4, \lambda}=\left[D_{\lambda}^{a} D_{x}^{b} w_{\lambda, n} \circ \psi_{\lambda, n}-D_{\lambda}^{a} D_{x}^{b} w_{\lambda, m} \circ \psi_{\lambda, m}\right] D_{\lambda}^{i_{1}} D_{x}^{j_{1}} \psi_{\lambda, m} \cdots D_{\lambda}^{i_{b}} D_{x}^{j_{b}} \psi_{\lambda, m}
$$

with $(a, b) \in \Sigma_{i, j}^{\prime}, i_{1}+\cdots+i_{b}=i-k-a, 0 \leq k \leq i-a$, and $j_{1}+\cdots+j_{b}=j$;

$$
\begin{aligned}
T_{5, \lambda}=D_{\lambda}^{a} D_{x}^{b} w_{\lambda, n}^{>} \circ \psi_{\lambda, n} & \\
& \quad\left[D_{\lambda}^{i_{1}} D_{x}^{j_{1}} \psi_{\lambda, n} \cdots D_{\lambda}^{i_{b}} D_{x}^{j_{b}} \psi_{\lambda, n}-D_{\lambda}^{i_{1}} D_{x}^{j_{1}} \psi_{\lambda, m} \cdots D_{\lambda}^{i_{b}} D_{x}^{j_{b}} \psi_{\lambda, m}\right]
\end{aligned}
$$

with $(a, b) \in \Sigma_{i, j}^{\prime}, i_{1}+\cdots+i_{b}=i-k-a, 0 \leq k \leq i-a$, and $j_{1}+\cdots+j_{b}=j$.

Before finishing the proof of Lemma 3.8, we state and prove the following lemma, which estimates the terms $T_{l}$ above.

Lemma 3.9. Assume the hypotheses of Lemma [3.8. Then, for every compact subset $G$ of $\Lambda \times B_{1}$ there exists a constant $C$ independent on $n$ such that, for $x \in G$ we have:

$$
\begin{aligned}
\left|T_{1, \lambda}(x)\right| & \leq C|x|^{L+1} \\
\left|T_{2, \lambda}(x)\right| & \leq C|x|^{(L-j+1)_{+}} \\
\left|T_{3, \lambda}(x)\right| & \leq C|x|^{(L-\tilde{\jmath}+1)_{+}}, \\
\left|T_{4, \lambda}(x)\right| & \leq C|x|^{(L-j+1)_{+}} \quad \text { in case that } m=0, \text { and } \\
\left|T_{5, \lambda}(x)\right| & \leq C|x|^{(L-j+1)_{+}} .
\end{aligned}
$$

Proof. For (53) we distinguish two cases. When $a+b+c<i_{0}+j_{0}$, then we use the mean value theorem and we bound $T_{1}$ by

$$
\left\|D_{\lambda}^{a} D_{1}^{c} D_{2}^{b+1} N_{2, \lambda}\right\|_{C^{0}}\left|w_{\lambda, n}(x)-w_{\lambda, m}(x)\right|\left|D_{\lambda}^{i_{1}} D_{x}^{j_{1}} w_{\lambda, m}(x) \cdots D_{\lambda}^{i_{b}} D_{x}^{j_{b}} w_{\lambda, m}(x)\right|,
$$


and we recall that

$$
\left|w_{\lambda, n}(x)-w_{\lambda, m}(x)\right|=\left|w_{\lambda, n}^{>}(x)-w_{\lambda, m}^{>}(x)\right| \leq\left\|w_{n}^{>}-w_{m}^{>}\right\|_{\Gamma\left(\Sigma_{i, j}^{\prime}, G^{*}\right)}|x|^{L+1} .
$$

In the second case when $a+b+c=i_{0}+j_{0}$, since $a+b+c \leq i+j$ then $j \geq i_{0}+j_{0}-i \geq j_{0} \geq L+1$ and thus $(L-j+1)_{+}=0$. Hence it is enough to see that $T_{1}$ is bounded, which follows immediately from hypothesis 2) of Proposition 3.7.

To bound $T_{2}$, we decompose the differences of (54) in telescopic form. Each difference will have a factor of the form

$$
\left|D_{\lambda}^{i_{l}} D_{x}^{j_{l}} w_{\lambda, n}(x)-D_{\lambda}^{i_{l}} D_{x}^{j_{l}} w_{\lambda, m}(x)\right| \leq\left\|w_{n}-w_{m}\right\|_{\Gamma\left(\Sigma_{i, j}^{\prime}, G^{*}\right)}|x|^{\left(L-j_{l}+1\right)_{+}},
$$

with $j_{l} \leq j$. The factor (58) is bounded by $C|x|^{(L-j+1)_{+}}$because $j_{l} \leq j$.

For $T_{3}$, we write

$$
\begin{aligned}
& D_{\lambda}^{\tilde{\imath}} D_{x}^{\tilde{\jmath}} \psi_{\lambda, n}-D_{\lambda}^{\tilde{\imath}} D_{x}^{\tilde{j}} \psi_{\lambda, m} \\
& =\sum_{(a, b, c) \in \sum_{i, \tilde{j}}^{* *}} \sum_{\substack{i_{1}+\ldots+i_{b}=\tilde{\imath}-a, j_{1}+\cdots+j_{b}=\bar{j}-c}} \\
& C\left[D_{\lambda}^{a} D_{1}^{c} D_{2}^{b} N_{1, \lambda} \circ\left(\mathrm{Id}, w_{\lambda, n}\right) D_{\lambda}^{i_{1}} D_{x}^{j_{1}} w_{\lambda, n} \cdots D_{\lambda}^{i_{b}} D_{x}^{j_{b}} w_{\lambda, n}\right. \\
& \left.-D_{\lambda}^{a} D_{1}^{c} D_{2}^{b} N_{1, \lambda} \circ\left(\mathrm{Id}, w_{\lambda, m}\right) D_{\lambda}^{i_{1}} D_{x}^{j_{1}} w_{\lambda, m} \cdots D_{\lambda}^{i_{b}} D_{x}^{j_{b}} w_{\lambda, m}\right] \\
& +\sum_{l=0}^{\tilde{i}} C D_{\lambda}^{l} B_{\lambda}\left[D_{\lambda}^{\tilde{\imath}-l} D_{x}^{\tilde{\jmath}} w_{\lambda, n}-D_{\lambda}^{\tilde{\imath}-l} D_{x}^{\tilde{\jmath}} w_{\lambda, m}\right] .
\end{aligned}
$$

We deduce the bound for $T_{3}$ claimed in Lemma 3.9 by applying the bounds for $T_{1}$ and $T_{2}$ with $i=\tilde{\imath}$ and $j=\tilde{\jmath}$ (with $N_{2}$ replaced by $N_{1}$ ).

To establish the bound for $T_{4}$, we take into account that $m=0$ and hence $w_{0}=w_{0}^{\leq}$. Note that $w_{0}$ is a polynomial and, therefore, it is sufficient to bound the first factor in the definition of $T_{4}$. For this we add and substract the term $D_{\lambda}^{a} D_{x}^{b} w_{\lambda, 0} \circ \psi_{\lambda, n}(x)$, and we use that $w_{\lambda, n}-w_{\lambda, 0}=w_{\lambda, n}-w_{\lambda}^{\leq}=w_{\lambda, n}^{>}$. We obtain the bound

$$
\begin{aligned}
& \left|D_{\lambda}^{a} D_{x}^{b} w_{\lambda, n}^{>} \circ \psi_{\lambda, n}(x)\right|+\left|D_{\lambda}^{a} D_{x}^{b} w_{\lambda, 0} \circ \psi_{\lambda, n}(x)-D_{\lambda}^{a} D_{x}^{b} w_{\lambda, 0} \circ \psi_{\lambda, 0}(x)\right| \\
& \quad \leq\left\|D_{\lambda}^{a} D_{x}^{b} w_{n}^{>}\right\|_{\Gamma\left(\Sigma_{i, j}^{\prime}, G^{*}\right)}\left|\psi_{\lambda, n}(x)\right|^{(L-b+1)_{+}} \\
& \quad+\left\|D_{\lambda}^{a} D_{x}^{b+1} w_{0}\right\|_{C^{0}}\left|\psi_{\lambda, n}(x)-\psi_{\lambda, 0}(x)\right| \\
& \quad \leq\left(\left\|A_{1, \lambda}\right\|+\varepsilon\right)^{(L-b+1)_{+}}\left\|D_{\lambda}^{a} D_{x}^{b} w_{n}^{>}\right\|_{\Gamma\left(\Sigma_{i, j}^{\prime}, G^{*}\right)}|x|^{(L-b+1)_{+}}+C|x|^{L+1},
\end{aligned}
$$

where we have used the previous bound for $T_{3}$.

Moreover, the second factor of $T_{4}, D_{\lambda}^{i_{1}} D_{x}^{j_{1}} \psi_{m} \cdots D_{\lambda}^{i_{b}} D_{x}^{j_{b}} \psi_{m}$, is such that $j_{1}+\cdots+j_{b}=j$ and hence it must have at least $(b-j)_{+}$derivatives with index $j_{l}=0$. Therefore by (49) this factor is bounded by $C|x|^{(b-j)_{+}}$.

For $T_{5}$, decomposing the difference

$$
D_{\lambda}^{i_{1}} D_{x}^{j_{1}} \psi_{n} \cdots D_{\lambda}^{i_{b}} D_{x}^{j_{b}} \psi_{n}-D_{\lambda}^{i_{1}} D_{x}^{j_{1}} \psi_{m} \cdots D_{\lambda}^{i_{b}} D_{x}^{j_{b}} \psi_{m}
$$


in a telescopic sum, each term will have a factor

$$
\left|D_{\lambda}^{i_{l}} D_{x}^{j_{l}} \psi_{\lambda, n}(x)-D_{\lambda}^{i_{l}} D_{x}^{j_{l}} \psi_{\lambda, m}(x)\right|
$$

which is bounded by $C|x|^{\left(L-j_{l}+1\right)_{+}} \leq C|x|^{(L-j+1)_{+}}$by the bounds for $T_{3}$.

Proof of Lemma 3.8 (continued). Using the estimates of Lemma 3.9 and (51), one easily checks that $\mathcal{B}_{n} \in \Gamma_{i, j}^{*}\left(G^{*}\right)$ and that there exists a constant $M$ independent on $n$ such that

$$
\left|\mathcal{B}_{n}(\lambda, x)\right| \leq M|x|^{(L-j+1)_{+}} .
$$

The same estimates work in the case $n=\infty$.

To establish c) of Lemma 3.8, from the definition of $\mathcal{A}_{n}$ and $\mathcal{B}_{n}$ we write

$$
D_{\lambda}^{i} D_{x}^{j} w_{n+1}^{>}=\mathcal{A}_{n} D_{\lambda}^{i} D_{x}^{j} w_{n}^{>}+\mathcal{B}_{n} .
$$

Then

$$
\left\|D_{\lambda}^{i} D_{x}^{j} w_{n+1}^{>}\right\|_{\Gamma_{i, j}^{*}\left(G^{*}\right)} \leq\left\|\mathcal{A}_{n}\right\|_{Y}\left\|D_{\lambda}^{i} D_{x}^{j} w_{n}^{>}\right\|_{\Gamma_{i, j}^{*}\left(G^{*}\right)}+\left\|\mathcal{B}_{n}\right\|_{\Gamma_{i, j}^{*}\left(G^{*}\right)} .
$$

Iterating this inequality, using that $\left\|\mathcal{A}_{n}\right\|$ and $\left\|\mathcal{B}_{n}\right\|$ are uniformly bounded, and $\left\|\mathcal{A}_{n}\right\| \leq \gamma<1$, we conclude that c) holds with $M_{i, j}=M /(1-\gamma)$.

Next we prove d) of Lemma 3.8. Given a compact set $G$ of $\Lambda \times B_{1}$, we need to prove that $\mathcal{B}_{n} \rightarrow \mathcal{B}_{\infty}$ converges uniformly on $G$. For this, we consider the differences (52) with $m=\infty$ in the larger compact set $G^{*}$. Examining the terms $T_{1}$ to $T_{5}$ in the analogous way as in Lemma 3.9, we see that all terms are bounded by factors which are estimated uniformly in $n$, times one of the following terms:

$$
\begin{gathered}
\left\|w_{n}^{>}-w_{m}^{>}\right\|_{\Gamma\left(\Sigma_{i, j}^{\prime}, G^{*}\right)} \\
\left|D_{\lambda}^{a} D_{1}^{c} D_{2}^{b} N_{j} \circ\left(x, w_{\lambda, n}(x)\right)-D_{\lambda}^{a} D_{1}^{c} D_{2}^{b} N_{j} \circ\left(x, w_{\lambda, \infty}(x)\right)\right| \\
\left\|D_{\lambda}^{a} D_{x}^{b} w_{\lambda, \infty} \circ \psi_{\lambda, n}-D_{\lambda}^{a} D_{x}^{b} w_{\lambda, \infty} \circ \psi_{\lambda, \infty}\right\|
\end{gathered}
$$

and

$$
\left\|D_{\lambda}^{a} D_{x}^{b} w_{\lambda, n} \circ \psi_{\lambda, n}-D_{\lambda}^{a} D_{x}^{b} w_{\lambda, \infty} \circ \psi_{\lambda, n}\right\| .
$$

The terms (60), (61) and (62) go to zero since $D_{\lambda}^{a} D_{1}^{c} D_{2}^{b} N_{j}$ and $D_{\lambda}^{a} D_{x}^{b} w_{\infty}$ are continuous, and since the sets $\left\{\left(\lambda, x, w_{\lambda, \infty}(x)\right) \mid(\lambda, x) \in G^{*}\right\}$ and $\left\{\left(\lambda, \psi_{\lambda, m}(x)\right) \mid\right.$ $\left.(\lambda, x) \in G^{*}, m \in \mathbb{Z}^{+} \cup\{\infty\}\right\}$ are compact.

Proof of Proposition 3.7. If we denote

$$
v_{n}=D_{\lambda}^{i} D_{x}^{j} w_{n}^{>},
$$

using (46) and the definitions of $w_{n}^{>}, \mathcal{A}_{n}$ and $\mathcal{B}_{n}$, we have

$$
\begin{aligned}
v_{0} & =0, \\
v_{n+1} & =\mathcal{A}_{n} v_{n}+\mathcal{B}_{n}, \quad n \geq 0 .
\end{aligned}
$$

Hence we can write

$$
v_{n+1}=\mathcal{B}_{n}+\mathcal{A}_{n} \mathcal{B}_{n-1}+\cdots+\mathcal{A}_{n} \mathcal{A}_{n-1} \cdots \mathcal{A}_{1} \mathcal{B}_{0} .
$$


We claim that $v_{n}$ converges to

$$
v_{\infty}=\mathcal{B}_{\infty}+\mathcal{A}_{\infty} \mathcal{B}_{\infty}+\mathcal{A}_{\infty}^{2} \mathcal{B}_{\infty}+\ldots
$$

uniformly on compact sets. Indeed

$$
\begin{aligned}
\mathcal{A}_{n} & \mathcal{A}_{n-1} \cdots \mathcal{A}_{n-k} \mathcal{B}_{n-k-1}-\mathcal{A}_{\infty}^{k+1} \mathcal{B}_{\infty} \\
= & \mathcal{A}_{n} \mathcal{A}_{n-1} \cdots \mathcal{A}_{n-k}\left(\mathcal{B}_{n-k-1}-\mathcal{B}_{\infty}\right)+\mathcal{A}_{n} \mathcal{A}_{n-1} \cdots \mathcal{A}_{n-k} \mathcal{B}_{\infty}-\mathcal{A}_{\infty}^{k+1} \mathcal{B}_{\infty} \\
= & \mathcal{A}_{n} \mathcal{A}_{n-1} \cdots \mathcal{A}_{n-k}\left(\mathcal{B}_{n-k-1}-\mathcal{B}_{\infty}\right) \\
\quad & +\sum_{i=1}^{k} \mathcal{A}_{n} \cdots \mathcal{A}_{n-i+1}\left(\mathcal{A}_{n-i}-\mathcal{A}_{\infty}\right) \mathcal{A}_{\infty}^{k-i} \mathcal{B}_{\infty} \\
& +\left(\mathcal{A}_{n}-\mathcal{A}_{\infty}\right) \mathcal{A}_{\infty}^{k} \mathcal{B}_{\infty}
\end{aligned}
$$

and, since given $g$ defined on $\Lambda \times B_{1}, \mathcal{A}_{n} g \rightarrow \mathcal{A}_{\infty} g$ uniformly on compact sets $G^{*}$ of $\Lambda \times B_{1}$, then

$$
\mathcal{A}_{n} \mathcal{A}_{n-1} \cdots \mathcal{A}_{n-k} \mathcal{B}_{n-k-1} \rightarrow \mathcal{A}_{\infty}^{k+1} \mathcal{B}_{\infty}
$$

uniformly on compact sets.

For $k<n$ we have

$$
\begin{gathered}
v_{n+1}(x)-\left(\mathcal{B}_{n}(x)+\mathcal{A}_{n} \mathcal{B}_{n-1}(x)+\cdots+\mathcal{A}_{n} \mathcal{A}_{n-1} \cdots \mathcal{A}_{n-k} \mathcal{B}_{n-k-1}(x)\right) \\
=\mathcal{A}_{n} \mathcal{A}_{n-1} \cdots \mathcal{A}_{n-k-1} \mathcal{B}_{n-k-2}(x)+\cdots+\mathcal{A}_{n} \mathcal{A}_{n-1} \cdots \mathcal{A}_{1} \mathcal{B}_{0}(x)
\end{gathered}
$$

and we can bound

$$
\begin{aligned}
& \left|v_{n+1}(x)-\left(\mathcal{B}_{n}(x)+\mathcal{A}_{n} \mathcal{B}_{n-1}(x)+\cdots+\mathcal{A}_{n} \mathcal{A}_{n-1} \cdots \mathcal{A}_{n-k} \mathcal{B}_{n-k-1}(x)\right)\right| \\
& \quad \leq \sum_{l=2}^{n-k} \gamma^{k+l}\left\|\mathcal{B}_{n-k-l}\right\|_{\Gamma_{i, j}^{*}\left(G^{*}\right)}|x|^{(L-j+1)_{+}} \\
& \quad \leq \frac{\gamma^{k+2}}{1-\gamma}|x|^{(L-j+1)_{+}} \sup _{0 \leq l \leq n-k}\left\|\mathcal{B}_{l}\right\|_{\Gamma_{i, j}^{*}\left(G^{*}\right)} .
\end{aligned}
$$

Taking the limit $n \rightarrow \infty$, we obtain

$$
\begin{aligned}
\mid v_{\infty}(x) & -\left(\mathcal{B}_{\infty}(x)+\mathcal{A}_{\infty} \mathcal{B}_{\infty}(x)+\cdots+\mathcal{A}_{\infty}^{k+1} \mathcal{B}_{\infty}(x)\right) \mid \\
\leq & \frac{\gamma^{k+2}}{1-\gamma} M|x|^{(L-j+1)_{+}} \sup _{l \geq 0}\left\|\mathcal{B}_{l}\right\|_{\Gamma_{i, j}^{*}\left(G^{*}\right)} .
\end{aligned}
$$

Then $D_{\lambda}^{i} D_{x}^{j} w_{n}^{>}$converges to

$$
v_{\infty}=\mathcal{B}_{\infty}+\mathcal{A}_{\infty} \mathcal{B}_{\infty}+\mathcal{A}_{\infty}^{2} \mathcal{B}_{\infty}+\ldots
$$

uniformly on compact sets $G^{*}$ as well as with the norm $\|\cdot\|_{\Gamma_{i, j}^{*}\left(G^{*}\right)}$.

Note that, since $\mathcal{A}_{\infty}$ is a contraction in $\Gamma_{i, j}^{*}\left(G^{*}\right)$ and $\mathcal{B}_{\infty}$ is in $\Gamma_{i, j}^{*}\left(G^{*}\right)$, then the resulting function $v_{\infty}$ is in $\Gamma_{i, j}^{*}\left(G^{*}\right)$. Moreover $\mathcal{B}_{\infty}$ is continuous. Then $v_{\infty}$ is the only solution of

$$
u=\mathcal{A}_{\infty} u+\mathcal{B}_{\infty},
$$


and hence $v_{\infty}$ is continuous.

For $1 \leq j \leq i_{0}-i+j_{0}$, we now show that the $D_{x}$ derivative of $D_{\lambda}^{i} D_{x}^{j-1} w_{n}^{>}$ converges uniformly to $v_{\infty}$ on compact sets of $\Lambda \times B_{1}$.

Indeed, for every $n$ we have

$$
D_{\lambda}^{i} D_{x}^{j-1} w_{\lambda, n}^{>}(y)-D_{\lambda}^{i} D_{x}^{j-1} w_{\lambda, n}^{>}(x)=\int_{0}^{1} d t D_{\lambda}^{i} D_{x}^{j} w_{\lambda, n}^{>}(x+t(y-x))(y-x)
$$

Passing to the limit, using that $D_{\lambda}^{i} D_{x}^{j} w_{n}^{>}$converges to $v_{\infty}$ in the compact set $G^{*}$ with $G=\{x+t(y-x) \mid t \in[0,1]\}$, we get

$$
D_{\lambda}^{i} D_{x}^{j-1} w_{\mu, \infty}^{>}(x)-D_{\lambda}^{i} D_{x}^{j-1} w_{\lambda, \infty}^{>}(x)=\int_{0}^{1} d t v_{\lambda, \infty}(x+t(y-x))(y-x) .
$$

Finally, using that $v_{\infty}$ is continuous, we conclude that $v_{\infty}$ is indeed $D_{\lambda}^{i} D_{x}^{j} w^{>}$.

If $j=0$ and $i \geq 1$, we show that the $D_{\lambda}$ derivative of $D_{\lambda}^{i-1} D_{x}^{j} w_{n}^{>}$converges uniformly to $v_{\infty}$ on compact sets of $\Lambda \times B_{1}$.

Indeed, from the identity

$$
D_{\lambda}^{i-1} D_{x}^{j} w_{\mu, n}^{>}(x)-D_{\lambda}^{i-1} D_{x}^{j} w_{\lambda, n}^{>}(x)=\int_{0}^{1} d t D_{\lambda}^{i} D_{x}^{j} w_{\lambda+t(\mu-\lambda), n}^{>}(x)(\mu-\lambda),
$$

we pass to the limit to obtain

$$
D_{\lambda}^{i-1} D_{x}^{j} w_{\mu, \infty}^{>}(x)-D_{\lambda}^{i-1} D_{x}^{j} w_{\lambda, \infty}^{>}(x)=\int_{0}^{1} d t v_{\lambda+t(\mu-\lambda), \infty}(x)(\mu-\lambda),
$$

and the argument finishes as before. This ends the proof of Proposition 3.7.

Now, we are in a position to complete the proof of Theorem 3.1.

Proof of Theorem 3.1. Since $F \in C^{\Sigma_{i, j}}$ for fixed $\lambda$, as a function of $x$ we have that $F_{\lambda}(\cdot) \in C^{i+j}$.

From Proposition 4.3 in [CFतll(0) (applied with $r$ replaced by $i+j$ ), we know that $w_{\lambda}^{>}=\lim _{n} \mathcal{M}^{n}(0)$, where the limit is obtained with $\lambda$ fixed and in the topology given in the space $\Gamma_{i+j-1, L}$ used in [CFतIIC0], and that this limit is $C^{i+j}$ as a function of $x$. Moreover, the limits are uniform in $\lambda$.

As a consequence, for every $k \leq i+j-1$ we have that

$$
\sup _{\Lambda \times B_{1}} \frac{\left|D_{x}^{k} w_{\lambda, n}^{>}(x)-D_{x}^{k} w_{\lambda, \infty}^{>}(x)\right|}{|x|^{(L-j+1)_{+}}} \leq C\left\|w_{\lambda, n}^{>}-w_{\lambda, \infty}^{>}\right\|_{\Gamma_{i+j-1, L}} \longrightarrow 0
$$

as $n$ tends to $\infty$.

We are now in position to apply Proposition 3.7 repeatedly. We first apply it with $i_{0}=0$ and $j_{0}=i+j$. We then apply it repeatedly from $i_{0}=1$ to $i_{0}=i$, where for each $i_{0}$ we apply the proposition starting with $j_{0}=0$ and then up to $j_{0}=i+j-i_{0}$.

\section{ACKNOWLEDGMENTS}

This work has been supported by the Comisión Conjunta Hispano Norteamericana de Cooperación Científica y Tecnológica. The final version was prepared while Rafael de la Llave was enjoying a Cátedra de la Fundación FBBV. 
X.C. was partially supported by the Spanish CICYT grant PB98-0932-C02-01, and by a Harrington Faculty Fellowship at the University of Texas-Austin. E.F. acknowledges the partial support of the the Spanish Grant DGICYT BFM20000805, the Catalan grant CIRIT 2000SGR-00027 and the INTAS project 00-221. The work of R.L. has been supported by NSF grants. R.L. thanks Universitat de Barcelona and Universitat Politècnica de Catalunya for hospitality during several visits.

\section{REFERENCES}

[CFdlL00] Xavier Cabré, Ernest Fontich, and Rafael de la Llave. The parameterization method for invariant manifolds I: manifolds associated to non-resonant subspaces. Preprint, 2000.

[CFdlL02] Xavier Cabré, Ernest Fontich, and Rafael de la Llave. The parameterization method for invariant manifolds III: overview and applications. In preparation, 2002.

[dlLMM86] Rafael de la Llave, Jose Manuel Marco, and Roberto Moriyón. Canonical perturbation theory of Anosov systems and regularity results for the Livšic cohomology equation. Ann. of Math. (2), 123(3):537-611, 1986.

Department of Mathematics, RLM 8.100, The University of Texas at Austin, Austin, TX 78712-1082, U.S.A,

E-mail address, X. Cabré: cabre@math.utexas.edu

Departament de Matemàtica Aplicada i Anàlisi, Universitat de Barcelona, Gran Via, 585, 08007 Barcelona, Spain

E-mail address, E. Fontich: fontich@mat.ub.es

Department of Mathematics, RLM 8.100, The University of Texas at Austin, Austin, TX 78712-1082, U.S.A,

E-mail address, R. de la Llave: llave@math.utexas.edu 